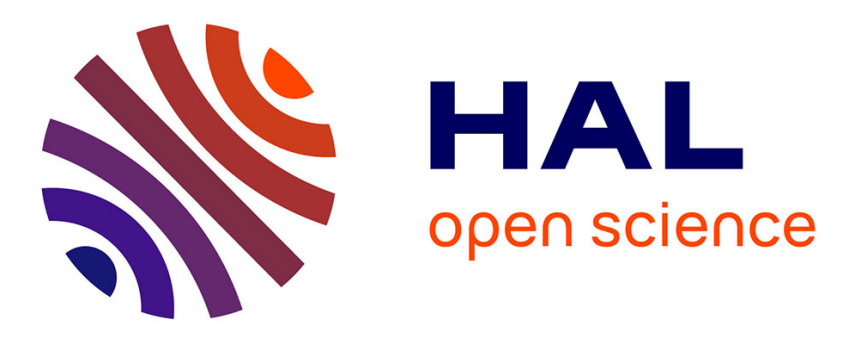

\title{
Complex Amplitudes Tracking Loop for multipath channel estimation in OFDM systems over slow to moderate fading
}

\author{
Laurent Ros, Hussein Hijazi, Eric Pierre Simon
}

\section{To cite this version:}

Laurent Ros, Hussein Hijazi, Eric Pierre Simon. Complex Amplitudes Tracking Loop for multipath channel estimation in OFDM systems over slow to moderate fading. Signal Processing, 2014, 97 (April), pp.134-145. 10.1016/j.sigpro.2013.10.014 . hal-00916576

\section{HAL Id: hal-00916576 https://hal.science/hal-00916576}

Submitted on 10 Dec 2013

HAL is a multi-disciplinary open access archive for the deposit and dissemination of scientific research documents, whether they are published or not. The documents may come from teaching and research institutions in France or abroad, or from public or private research centers.
L'archive ouverte pluridisciplinaire HAL, est destinée au dépôt et à la diffusion de documents scientifiques de niveau recherche, publiés ou non, émanant des établissements d'enseignement et de recherche français ou étrangers, des laboratoires publics ou privés. 


\title{
Complex Amplitudes Tracking Loop for multipath channel estimation in OFDM systems over slow to moderate fading ${ }^{\text {tr }}$
}

\author{
Laurent ROS, Hussein HIJAZI and Eric-Pierre SIMON, th th
}

\begin{abstract}
This paper deals with multipath channel estimation for Orthogonal Frequency-Division Multiplexing systems under slow to moderate fading conditions. Most of the conventional methods exploit only the frequency-domain correlation by estimating the channel at pilot frequencies, and then interpolating the channel frequency response. More advanced algorithms exploit in addition the time-domain correlation, by employing Kalman filters based on the approximation of the time-varying channel. Adopting a parametric approach and assuming a primary acquisition of the path delays, channel estimators have to track the complex amplitudes of the paths. In this perspective, we propose a less complex algorithm than the Kalman methods, inspired by second-order Phase-Locked Loops. An error signal is created from the pilot-aided Least-Squares estimates of the complex amplitudes, and is integrated by the loop to carry out the final estimates. We derive closed-form expressions of the mean squared error of the algorithm and of the optimal loop coefficients versus the channel state, assuming a Rayleigh channel with Jakes'Doppler spectrum. The efficiency of our reduced complexity algorithm is demonstrated, with an asymptotic mean squared error lower than the first-order auto-regressive Kalman filters reported in the literature, and almost the same as a second-order Kalman-based algorithm.
\end{abstract}

\footnotetext{
${ }^{2}$ Part of this work was presented in the conference ISCCSP 2010 [1]. This paper is an improved version of the on-line (unpublished) Gipsa-lab research report [2]

L. Ros is with GIPSA-lab, Image and Signal Department, BP 46 - 38402 Saint Martin d'Hères FRANCE (e-mail corresponding author: laurent.ros@gipsa-lab.grenoble-inp.fr). H. Hijazi is with Lebanese International University (LIU), Beirut, LEBANON (hijazi.hussein@liu.edu.lb). E.P. Simon is with IEMN lab, TELICE group, 59655 Villeneuve d'Ascq, University of Lille, FRANCE (eric.simon@univ-lille1.fr)
} 
Keywords- OFDM, Channel estimation, Rayleigh multipath channel with Jakes' Doppler spectrum, Phase-Locked Loop (PLL), Kalman filter (KF), second-order model.

\section{Introduction}

Orthogonal Frequency-Division Multiplexing (OFDM) is an effective technique for alleviating frequency-selective channel effects in wireless communication systems. In this technique, a wideband frequency-selective channel is converted to a number of parallel narrow-band flat fading subchannels which are free of Inter-Symbol-Interference (ISI) and free of Inter-Carrier Interference (ICI) (for negligible channel time variation within one OFDM symbol period $T$ ). For coherent detection of the information symbols, reliable estimation of the gain of each subchannel in the OFDM system is crucial.

\subsection{Some approaches to channel estimation in OFDM}

Most of the conventional methods work in a symbol-by-symbol scheme [3, 4, 5] by using the correlation of the channel only in the frequency-domain (FD), i.e. the correlation between subchannels. Generally, they consist in estimating the channel at the pilot subcarrier position and then interpolating it over the entire frequency grid [3]. The channel estimation at the pilot frequencies can be based on the Least-Squares (LS) criterion, or, for better performance, on the Linear-Minimum-Mean-Square-Error (LMMSE) criterion [4]. In [5], Low-Pass Interpolation (LPI) has been shown to perform better than all interpolation techniques used in channel estimation. This channel estimator will be called conventional LS(FD)-LPI in this paper.

Though the conventional methods can operate with time-varying channels, the information of the time-domain correlation is not exploited. However, the channel estimation process can be theoretically greatly improved by using the previous OFDM symbols, according to the on-line Bayesian Cramer-Rao Bound (BCRB) analysis in [6]. Thus, Chen and Zhang proposed in [7] a structure to track the complex gains of each subchannel by using one Kalman filter (KF) per sub-channel. In practice, only a subset of pilot-subcarriers is used to perform the per-subchannel KF, and the global frequency response of the channel is still obtained by LPI interpolation. This estimator will be named Kalman(FD)-LPI in the paper. Other works still exploit time 
and frequency correlation for OFDM channel estimation by using additional assumptions or different approaches. Assuming the availability of the power delay profile, a data-aided KF estimator (derived from the Expectation-Maximization algorithm framework) is employed in [8] to track the discrete-time impulse response of the channel (i.e in Time-Domain (TD)). And a low-complexity parameter reduction approach based on the eigenvalue decomposition of the auto-correlation matrix of the channel (in FD) is proposed in [9]. It tracks the channel coefficients in the dominant eigenvectors subspace basis by KF, and then performs eigenvalues interpolation to compute the channel frequency response. This estimator will be denoted Kalman-EIG in this paper.

In the same context of reducing the signal subspace dimension, we now focus on the class of parametric channel estimators. Assuming a multipath channel structure, estimation can be reduced to the estimation of certain physical propagation parameters, such as multipath delays and multipath Complex Amplitudes (CAs) [10, 11, 12, 13, 14]. It is well known that in wireless radio channels, the delays are quasi-constant over a large number of symbols. Consequently, the number of paths and path delays can be very accurately estimated, for example by applying the MDL (Maximum Description Length) principle combined with the ESPRIT (Estimation of Signal Parameters by Rotational Invariance Techniques) method as proposed in [10], and adopted many times $[11,13,14])$. Several papers on OFDM channel estimation focus their works on the crucial CAs tracking problem, assuming the delays are invariant and perfectly estimated. This approach will be adopted in this paper. In $[13,14]$ we have addressed this issue for the special case of fast time-varying channel (i.e. with normalized Doppler spread $f_{d} T \geq 10^{-2}$ ), by using polynomial modeling of the CA time-variation. We have also addressed it in [15] for the joint carrier frequency offset and high speed channel estimation problem.

\subsection{Motivation of the work and contributions}

Second-order versus first-order algorithms. the use of KF for channel estimation has received great attention in recent years in the wireless communication literature. It is true for most systems, e.g. MIMO [16, 17] or single-carrier systems [18, 19], as well as in OFDM systems, as mentioned before [7, 8, 9, 14]. All the aforementioned 
works based their KF on the AR approximation of the widely accepted Rayleigh fading channel with the Jakes' Doppler spectrum [20], called the "Rayleigh-Jakes" model in this paper, as developed in [21]. The first-order Gauss-Markov assumption (AR1 model) is most often retained $[8,9,16,17,18,19,22,23]$. The so-called AR1-Kalman estimators are convenient for the very high mobility case, leading to quasi-optimal performance, as seen, for example, in $[14,15]$. In these works an AR1-Kalman is actually used to track the polynomial Basis Expansion Model coefficients of the high speed channel. However, in the more common scenario of slow to moderate fading with negligible variation during one symbol (i.e. $f_{d} T \leq 10^{-2}$, as in $[7,8,16,17,18,19]$ ), the AR1-Kalman estimators of the literature seem to exhibit poor performance compared to BCRB lower bounds, as seen in [1]-Fig.10. On the other hand, [24] shows, in a single-carrier single-path context, that the MSE performance of a KF can be well improved by switching from the AR1 to a second-order model (of the integrated random walk (IRW) model type) for the approximation of the Jakes'process. Indeed, for low $f_{d} T$, the exact channel CA continues in a given direction during several symbols, and a second-order approximation model can generally take into account this strong trend behaviour better than a first-order model $[25,26]$.

Reduced complexity algorithms compared to Kalman. KF-based algorithms require the updating of the coefficients of the algorithm at each iteration (each new OFDM symbol), and are quite complex as a result. However, reduced complexity adaptive algorithms can be obtained, using constant coefficients. They can be designed, if an a priori model of the dynamic of time-varying parameters (i.e. hypermodel) is available, such as a Wiener LMS adaptation algorithm ([26]), or as a steady-state version of the $\mathrm{KF}$, since a time-varying KF becomes a time-invariant filter after convergence, see [27], ch 13.5. Such algorithms are generally slower than the KF during the convergence, but can have the same asymptotic performance in tracking mode. In this family, the classical Least-Mean-Squared (LMS) algorithm can be regarded as a steady-state version of 
a KF based on a first-order random-walk (RW) model. Second-order channel tracking algorithms described as predictive LMS, or as a steady-state version of an IRW modelbased KF, have also been proposed in [25, 26, 28]. However, they have been developed for the single-carrier transmission, and without simple closed-form formulas versus the channel state for the tuning and performance of the estimators.

Approach and contributions. in this paper, we propose and analyze a low-complexity on-line recursive algorithm with constant coefficients for the multipath CAs estimation problem under the common slow to moderate channel variations scenario $\left(f_{d} T \leq\right.$ $10^{-2}$ ). It is developed for OFDM systems with comb-type pilots within the framework of parametric channel estimators, exploiting the availability of delay related information (assuming a primary acquisition as in $[10,13,14,15])$ for tracking the CA variations. The proposed algorithm is based on a Complex Amplitudes Tracking Loop (CATL) structure. This structure is inspired by second-order digital Phase-Locked Loops (PLL) [29, 30], as well as by the "prediction-correction" principle of the KF (in the steady-state mode) given the close link between the two ([31, 32]). The error signal that feeds the loop is based on the LS estimate of the paths CA, obtained for each current symbol from the pilot-subcarriers. The proposed LS-CATL algorithm can be seen as an extension for the multipath OFDM case of the second-order adaptive algorithms of [25, 26](and also [24]-ch 4.1), using the parametric estimation framework. Our main contributions can be summarized below:

- proposition, interpretation, and analytical optimization of a simple on-line secondorder (multipath) CAs tracking algorithm with almost the same asymptotic MSE performance as a second-order KF derived with the same assumptions (parametric modeling and a priori knowledge), but with a reduced complexity,

- derivation of closed-form expressions usable to tune the coefficients of the CATL as well as to predict the MSE performance with respect to the channel state (Doppler spread, power-delay profile, SNR) under "Rayleigh-Jakes" assumption. 
- evaluation of the benefit of the second-order proposed algorithm compared to first-order KF-based reference algorithms or other conventional (FD interpolation) methods of the literature, for the common slow to moderate fading channel. The paper is organized as follows: Section II describes the system model and objectives. Section III derives the proposed algorithm and its analysis, and the different results are discussed in Section IV.

Notations: $[\mathbf{x}]_{k}$ denotes the $k$ th entry of vector $\mathbf{x}$, and $[\mathbf{X}]_{k, m}$ the $[k, m]$ th entry of matrix $\mathbf{X}$ (indices begin from 1). $\mathbf{I}_{N}$ is an $N \times N$ identity matrix. The notation $\operatorname{diag}\{\mathbf{x}\}$ stands for a diagonal matrix with $\mathbf{x}$ on its diagonal, $\operatorname{diag}\{\mathbf{X}\}$ is a vector whose elements are the elements of the diagonal of $\mathbf{X}$, and blkdiag $\{\mathbf{X}, \mathbf{Y}\}$ is a block diagonal matrix with the matrices $\mathbf{X}$ and $\mathbf{Y}$ on its diagonal. The superscripts $(\cdot)^{T},(\cdot)^{H},|\cdot|$, and $\operatorname{Tr}(\cdot)$ respectively stand for transpose and Hermitian operators, determinant and trace operations. $J_{0}(\cdot)$ is the zeroth-order Bessel function of the first kind.

\section{System Model}

\subsection{OFDM Transmission over multipath channel}

Let us consider an OFDM system with $N$ subcarriers, and a cyclic prefix length $N_{g}$. The duration of an OFDM symbol is $T=v T_{s}$, where $T_{s}$ is the sampling time and $v=N+N_{g}$. Let $\mathbf{x}_{(n)}=\left[x_{(n)}\left[-\frac{N}{2}\right], x_{(n)}\left[-\frac{N}{2}+1\right], \ldots, x_{(n)}\left[\frac{N}{2}-1\right]\right]^{T}$ be the vector containing the $N$ QAM symbols for the $n$th OFDM symbol. After transmission over a multipath channel and FFT demodulation, the observation is given by [10, 13]:

$$
\mathbf{y}_{(n)}=\mathbf{H}_{(n)} \mathbf{x}_{(n)}+\mathbf{w}_{(n)}
$$

where $\mathbf{w}_{(n)}$ is a $N \times 1$ zero-mean complex circular Gaussian noise vector with covariance matrix $\sigma^{2} \mathbf{I}_{N}$, and $\mathbf{H}_{(n)}$ is a $N \times N$ diagonal matrix with diagonal elements

$$
\left[\mathbf{H}_{(n)}\right]_{k, k}=\frac{1}{N} \sum_{l=1}^{L}\left[\alpha_{l(n)} \times e^{-j 2 \pi\left(\frac{k-1}{N}-\frac{1}{2}\right) \tau_{l}}\right]
$$


$L$ is the total number of propagation paths, $\left\{\alpha_{l(n)}\right\}$ is the $l$ th CA at $n$th OFDM symbol with variance $\sigma_{\alpha_{l}}^{2}$ (with $\sum_{l=1}^{L} \sigma_{\alpha_{l}}^{2}=1$ ), and $\tau_{l} \times T_{s}$ is the $l$ th delay ( $\tau_{l}$ is not necessarily an integer, but $\tau_{L}<N_{g}$ ). The $L$ individual elements of $\left\{\alpha_{l(n)}\right\}$ are uncorrelated with respect to each other. Using (2), the observation model (1) can be re-written [10] as

$$
\mathbf{y}_{(n)}=\operatorname{diag}\left\{\mathbf{x}_{(n)}\right\} \mathbf{F} \boldsymbol{\alpha}_{(n)}+\mathbf{w}_{(n)}
$$

where $\boldsymbol{\alpha}_{(n)}=\left[\alpha_{1(n)}, \ldots, \alpha_{L(n)}\right]^{T}$ and $\mathbf{F}$ is an $N \times L$ Fourier matrix depending on the delay distribution, with elements given by $[\mathbf{F}]_{k, l}=e^{-j 2 \pi\left(\frac{k-1}{N}-\frac{1}{2}\right) \tau_{l}}$.

We assume the "Rayleigh-Jakes" model [20] for the channel, with Doppler frequency $f_{d}$. It means the $L$ CAs $\alpha_{l(n)}$ are independent wide-sense stationary zero-mean complex circular Gaussian processes, with correlation coefficients for a time-lag $k$ given by

$$
\mathrm{R}_{\alpha_{l}}^{(k)}=\mathrm{E}\left[\alpha_{l(n)} \alpha_{l(n-k)}{ }^{H}\right]=\sigma_{\alpha_{l}}^{2} \cdot J_{0}\left(2 \pi f_{d} T k\right)
$$

\subsection{Pilot Pattern}

The $N_{p}$ pilot subcarriers are evenly inserted into the $N$ subcarriers at the positions $\mathcal{P}=\left\{p_{s} \mid p_{s}=(s-1) L_{f}+1, s=1, \ldots, N_{p}\right\}$ with $L_{f}$ the distance between two adjacent pilots. The received pilot subcarriers can be written as

$$
\mathbf{y}_{\mathbf{p}(n)}=\mathcal{K}_{(n)} \boldsymbol{\alpha}_{(n)}+\mathbf{w}_{\mathbf{p}(n)}
$$

where $\mathbf{y}_{\mathbf{p}}$ and $\mathbf{w}_{\mathbf{p}}$ are $N_{p} \times 1$ vectors. The $N_{p} \times L$ matrix $\mathcal{K}_{(n)}$ is defined by

$$
\mathcal{K}_{(n)}=\operatorname{diag}\left\{\mathbf{x}_{\mathbf{p}(n)}\right\} \mathbf{F}_{\mathbf{p}}
$$


Note that $\mathcal{K}_{(n)}$ is computed for each OFDM symbol, using knowledge of the $N_{p} \times 1$ data pilot vector $\mathbf{x}_{\mathbf{p}(n)}$ and the delays $\left\{\tau_{l}\right\}$ through the $N_{p} \times L$ matrix $\mathbf{F}_{\mathbf{p}}$ with elements

$$
\left[\mathbf{F}_{\mathbf{p}}\right]_{k, l}=e^{-j 2 \pi\left(\frac{p_{k}-1}{N}-\frac{1}{2}\right) \tau_{l}}
$$

\subsection{Estimation objectives}

We wish to estimate the CAs $\boldsymbol{\alpha}_{(n)}$ assuming the knowledge of pilots subcarriers $\mathbf{x}_{\mathbf{p}(n)}$ and delays $\boldsymbol{\tau}=\left[\tau_{1}, \ldots, \tau_{L}\right]^{T}$. The estimation is based on the observation model (5) that can be reformulated as $\mathbf{y}_{\mathbf{p}(n)}=\mathcal{K}\left(\mathbf{x}_{\mathbf{p}(n)}, \boldsymbol{\tau}\right) \boldsymbol{\alpha}_{(n)}+\mathbf{w}_{\mathbf{p}(n)}$. We restrict the problem to the on-line estimation, which means current and previous observations are available (i.e. for indices $n, n-1, n-2, \ldots)$ to estimate $\boldsymbol{\alpha}$ at time index $n$.

\section{Complex Amplitudes Tracking algorithm}

The proposed tracking algorithm, called the LS-CATL algorithm, is built from a general second-order recursive structure (CATL) presented below, and from a specific error signal (based on pilots and LS criterion) that will specify the error detector of the structure, presented subsequently.

\subsection{Structure of the algorithm: CA Tracking Loop}

The estimate of $\boldsymbol{\alpha}_{(n)}$, denoted $\hat{\boldsymbol{\alpha}}_{(n \mid n)}$, is updated at a symbol rate by the computation of an error signal $\boldsymbol{v}_{\boldsymbol{\epsilon}(n)}$, next filtered by a second-order feedback loop. The recursive equations of the second-order CATL, using a PLL-type formulation ([29, 30]), are:

$$
\begin{aligned}
\text { Error detector }: \boldsymbol{v}_{\boldsymbol{\epsilon}(n)} & =\text { function of }\left\{\mathbf{y}_{\mathbf{p}(n)} ; \hat{\boldsymbol{\alpha}}_{(n \mid n-1)}\right\} \\
\text { Final estimate }: \hat{\boldsymbol{\alpha}}_{(n \mid n)} & =\hat{\boldsymbol{\alpha}}_{(n \mid n-1)}+\mu_{1} \cdot \boldsymbol{v}_{\boldsymbol{\epsilon}(n)} \\
\text { Loop filter }: \boldsymbol{v}_{\mathbf{L a g}(n)} & =\boldsymbol{v}_{\mathbf{L a g}(n-1)}+\boldsymbol{v}_{\boldsymbol{\epsilon}(n)} \\
\boldsymbol{v}_{\boldsymbol{c}(n)} & =\mu_{1} \cdot \boldsymbol{v}_{\boldsymbol{\epsilon}(n)}+\mu_{2} \cdot \boldsymbol{v}_{\mathbf{L a g}(n)} \\
\text { NC Generator }: \hat{\boldsymbol{\alpha}}_{(n+1 \mid n)} & =\hat{\boldsymbol{\alpha}}_{(n \mid n-1)}+\boldsymbol{v}_{\boldsymbol{c}(n)}
\end{aligned}
$$




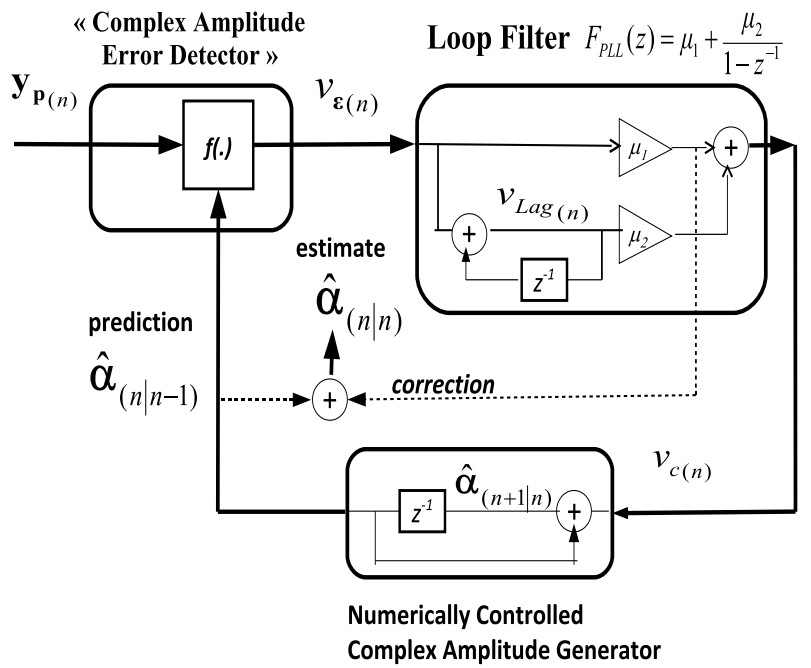

Figure 1: Structure of the second-order complex amplitude tracking loop, inspired by second-order digital PLL

where $\mu_{1}, \mu_{2}$ are the (real positive) loop coefficients. Based on these equations, the structure of the CATL is shown in Fig. 1. As in a second-order digital PLL [30], we find in cascade an error detector that delivers error signal $\boldsymbol{v}_{\boldsymbol{\epsilon}(n)}$, a proportional-integral (PI) loop filter $F_{P L L}(z)=\mu_{1}+\frac{\mu_{2}}{1-z^{-1}}$ (or lead-lag filter) parametrized by $\mu_{1}$ and $\mu_{2}$, and a Numerically Controlled (NC) Generator delivering the predicted estimates $\hat{\boldsymbol{\alpha}}_{(n \mid n-1)}$. However, the estimates are multiple complex amplitudes, instead of one real phase in a PLL (then delivered by a NC Oscillator). $\boldsymbol{v}_{\boldsymbol{\epsilon}(n)}$ is a complex vector in the output of a "Complex Amplitude Error Detector" (CAED) (vs a real scalar in the output of a phase error detector in a PLL), to be defined in (8) from the new measurement $\mathbf{y}_{\mathbf{p}(n)}$ and the prediction $\hat{\boldsymbol{\alpha}}_{(n \mid n-1)}$. Also, the final estimate $\hat{\boldsymbol{\alpha}}_{(n \mid n)}$ is not directly the prediction $\hat{\boldsymbol{\alpha}}_{(n \mid n-1)}$ as in conventional PLL, but is delivered after a correction step according to (9). Thus, an additional branch is added as a dotted line in Fig. 1.

Using (9), we can compact the second equation of the loop filter (11) and the NCG equation (12) by the unique equation (13): 


$$
\hat{\boldsymbol{\alpha}}_{(n+1 \mid n)}=\hat{\boldsymbol{\alpha}}_{(n \mid n)}+\boldsymbol{\mu}_{2} \cdot \boldsymbol{v}_{\mathbf{L a g}(n)}
$$

showing that the sum accumulator of the error signal weighted by $\mu_{2}$, i.e. $\mu_{2} . v_{\mathbf{L a g}(n)}$, is an estimate of the speed evolution (or slope) of $\boldsymbol{\alpha}$, useful to predict the CAs evolution.

\subsection{Error signal specific to the LS-CATL algorithm}

We now have to define an error signal vector in place of eq. (8). Inspired by PLL, a good candidate (among several possibilities [1]) is an error signal vector $\boldsymbol{v}_{\boldsymbol{\epsilon}(n)}$ collinear (in absence of noise) with the prediction error vector $\boldsymbol{\epsilon}_{\operatorname{Pred}(n)}=\boldsymbol{\alpha}_{(n)}-\hat{\boldsymbol{\alpha}}_{(n \mid n-1)}$, in order to get a detector, which is perfectly linear and free from inter-path-interference. In this perspective, let us first consider the LS-estimator of $\boldsymbol{\alpha}_{(n)}$ that permits, among all estimators $\hat{\boldsymbol{\alpha}}_{(n)}$, us to minimize the squares error $\left(\mathbf{y}_{\mathbf{p}(n)}-\mathcal{K}_{(n)} \hat{\boldsymbol{\alpha}}_{(n)}\right)^{H} \cdot\left(\mathbf{y}_{\mathbf{p}(n)}-\mathcal{K}_{(n)} \hat{\boldsymbol{\alpha}}_{(n)}\right)$ for the current OFDM symbol:

$$
\begin{aligned}
\boldsymbol{\alpha}_{\mathbf{L S}(n)} & =\mathbf{G}_{(n)} \mathbf{y}_{\mathbf{p}(n)} \\
\text { with } \quad \mathbf{G}_{(n)} & =\left(\mathcal{K}_{(n)}^{H} \mathcal{K}_{(n)}\right)^{-1} \mathcal{K}_{(n)}^{H}
\end{aligned}
$$

We see from (14)\&(15)\&(5) that the LS estimator is unbiased, with $\boldsymbol{\alpha}_{\mathbf{L S}(n)}=\boldsymbol{\alpha}_{(n)}+$ $\boldsymbol{N}_{(n)}$ where $\boldsymbol{N}_{(n)}$ is a zero-mean complex Gaussian noise vector. So, we propose to use simply the difference between the LS estimator $\boldsymbol{\alpha}_{\mathbf{L S}(n)}$ for the nth OFDM block and the prediction, $\hat{\boldsymbol{\alpha}}_{(n \mid n-1)}$, as an error signal vector in place of (8):

$$
\boldsymbol{v}_{\boldsymbol{\epsilon}(n)}=\mathbf{G}_{(n)} \mathbf{y}_{\mathbf{p}(n)}-\hat{\boldsymbol{\alpha}}_{(n \mid n-1)}
$$

Thus, this specific error signal vector (16) has a simple linear form versus the prediction error vector $\boldsymbol{\epsilon}_{\text {Pred }(n)}=\boldsymbol{\alpha}_{(n)}-\hat{\boldsymbol{\alpha}}_{(n \mid n-1)}$, as seen while using (14)\&(15)\&(5):

$$
\boldsymbol{v}_{\boldsymbol{\epsilon}(n)}=k_{d} \cdot\left\{\boldsymbol{\alpha}_{(n)}-\hat{\boldsymbol{\alpha}}_{(n \mid n-1)}\right\}+\boldsymbol{N}_{(n)}
$$

The real coefficient $k_{d}$ is the gain factor of the CAED, reduced here to $k_{d}=1$. And 
$\boldsymbol{N}_{(n)}=\left[N_{1(n)}, \ldots, N_{L(n)}\right]^{T}$ is the (temporally uncorrelated) zero-mean disturbance due to the additive thermal noise $\mathbf{w}_{\mathbf{p}(n)}$ in the input of the CAED, and represents the so-called (input) loop noise (i.e. in the input of the loop but in the output of the CAED). We have $\boldsymbol{N}_{(n)}=\mathbf{G}_{(n)} \mathbf{w}_{\mathbf{p}(n)}$, with a correlation matrix $E\left\{\boldsymbol{N}_{(n)} \cdot \boldsymbol{N}_{(n)}^{H}\right\}=\sigma^{2} \cdot\left(\mathbf{F}_{\mathbf{p}}^{H} \mathbf{F}_{\mathbf{p}}\right)^{-1}$, and a mean variance (per path, $\sigma_{N}^{2}=\frac{1}{L} \cdot \sum_{l=1}^{L} \sigma_{N_{l}}^{2}$ ):

$$
\begin{aligned}
\sigma_{N}^{2} & =\frac{\sigma^{2}}{N_{p}} \times \lambda_{N} \\
\text { with } \lambda_{N} & =\frac{1}{L} \cdot \operatorname{Tr}\left\{\left(\frac{1}{N_{p}} \cdot \mathbf{F}_{\mathbf{p}}^{H} \mathbf{F}_{\mathbf{p}}\right)^{-1}\right\} \geq 1
\end{aligned}
$$

where $N_{p}$ is the number of pilot subcarriers. The (input) loop noise variance is minimum (i.e. $\sigma_{N}^{2}(\min )=\frac{\sigma^{2}}{N_{p}}$ and $\lambda_{N}=1$ ) if $\boldsymbol{N}_{(n)}$ is uncorrelated from one path to another, i.e when $\mathbf{F}_{\mathbf{p}}^{H} \mathbf{F}_{\mathbf{p}}$ is a diagonal matrix. This condition depends on the delays distribution.

\subsection{Computational Complexity and comparison with reference KFs}

The CATL can be interpreted as a reduced complexity approach compared to reference KFs designed for the complete multi-carrier and multi-path observation model (5) such as presented in Appendix A (AR1-Kalman and Or2-Kalman). Indeed, the CATL acts in each branch l, i.e. for each multi-path component $\alpha_{l}$, as a "simplified" IRW model-based KF. Each KF is "simplified" in the sense it is designed for the simplified single-carrier and single-path scenario, as in [24], and it operates only in steady-state mode. But we have chosen an error signal (16) able to cope with the more complicated multi-carrier and multi-path scenario. This interpretation of the CATL structure is more detailed (i.e. derived from the equations) in Appendix B.

Let us determine now the implementation complexity in terms of the number of the complex multiplications needed for each OFDM symbol for our LS-CATL algorithm. The matrices $\mathbf{G}_{(n)}\left(\right.$ size $\left.L \times N_{p}\right)$ and $\mathcal{K}_{(n)}\left(\right.$ size $\left.N_{p} \times L\right)$ are assumed to be precomputed and stored, if the delays are invariant for a great number of OFDM symbols. Then, we just have $N_{p} \times L$ multiplications for the LS estimate (16) used in the error detector (8), 
plus $2 L$ multiplications (or just $L$ if $\mu_{2}=0$ ) in the loop filter (11). Table 1 compares this complexity to reference KFs presented in Appendix A. It is noteworthy that the LS-CATL algorithm is computationally less demanding technique than Kalman filters, since the latter require inversion of matrices of size $N_{p} \times N_{p}$ for the Kalman gain matrix computation (A.8), plus multiplications of matrix with size $N_{p} \times 2 L, 2 L \times 2 L$, or $N_{p} \times$ $N_{p}$ for the update of the filters coefficients (see (A.6)-(A.10)). The LS-CATL approach presents finally a linear complexity in terms of the number of pilot subcarriers $N_{p}$ $\left(O\left(N_{p}\right)\right)$ versus a cubic complexity $\left(O\left(N_{p}^{3}\right)\right)$ for the reference Kalman algorithms.

\begin{tabular}{cc}
\hline & Number of complex multiplications per OFDM symbol \\
\hline \hline 2nd-order LS-CATL & $N_{p} \times L+2 L$ \\
Or2-Kalman & $N_{p}^{3}+N_{p}^{2} \times 3 L+N_{p} \times\left(6 L^{2}+3 L\right)+4 L^{2}+2 L$ \\
\hline 1st-order LS-CATL & $N_{p} \times L+L$ \\
AR1-Kalman & $N_{p}^{3}+N_{p}^{2} \times 2 L+N_{p} \times\left(2 L^{2}+2 L\right)+L^{2}+L$ \\
\hline
\end{tabular}

Table 1: Complexity of the LS-CATL proposed algorithm (first-order and second-order versions) compared to reference KFs (AR1-Kalman and Or2-Kalman, described in Appendix A)

\subsection{General properties and theoretical MSE analysis}

\subsubsection{Second-order closed-loop transfer function}

The estimation error of the tracking algorithm is defined as

$$
\boldsymbol{\epsilon}_{(n)}=\boldsymbol{\alpha}_{(n)}-\hat{\boldsymbol{\alpha}}_{(n \mid n)}
$$

We want to obtain the transfer function between the true vector parameter and the estimate. Combining equations (13) and (9), we have:

$$
\hat{\boldsymbol{\alpha}}_{(n \mid n)}=\hat{\boldsymbol{\alpha}}_{(n-1 \mid n-1)}+\mu_{1} \cdot \boldsymbol{v}_{\boldsymbol{\epsilon}(n)}+\mu_{2} \cdot \boldsymbol{v}_{\mathbf{L a g}(n-1)}
$$

By using (10), the Z-domain transform of (21) leads to

$$
\hat{\boldsymbol{\alpha}}(z) \cdot\left[1-z^{-1}\right]=\left[\mu_{1}+\frac{\mu_{2} \cdot z^{-1}}{1-z^{-1}}\right] \cdot \boldsymbol{v}_{\varepsilon}(z)
$$


Combining the general loop equation (22) with the specific (LS-based) error signal (17) rewritten versus the estimation error as

$$
\boldsymbol{v}_{\boldsymbol{\epsilon}(n)}=\frac{k_{d}}{1-k_{d} \mu_{1}} \cdot\left\{\boldsymbol{\alpha}_{(n)}-\hat{\boldsymbol{\alpha}}_{(n \mid n)}\right\}+\frac{1}{1-k_{d} \mu_{1}} \cdot \boldsymbol{N}_{(n)}
$$

we obtain in the Z-transform domain:

$$
\hat{\boldsymbol{\alpha}}(z)=L(z) \cdot \boldsymbol{\alpha}(z)+\frac{L(z)}{k_{d}} \cdot \boldsymbol{N}(z)
$$

where $L(z)$ is the transfer function of the CATL defined by

$$
L(z)=\frac{\frac{k_{d}}{1-k_{d} \mu_{1}} F(z)}{\left(1-z^{-1}\right)+\frac{k_{d}}{1-k_{d} \mu_{1}} F(z)}
$$

with respect to $F(z)=\mu_{1}+\frac{\mu_{2} \cdot z^{-1}}{1-z^{-1}}$. Hence, the CATL transfer function can be written versus the loop coefficients $\left(\mu_{1}, \mu_{2}\right)$ as ${ }^{1}$

$$
L(z)=\frac{k_{d}\left[(z-1)^{2} \cdot \mu_{1}+(z-1) \cdot\left(\mu_{1}+\mu_{2}\right)+\mu_{2}\right]}{(z-1)^{2}+(z-1) \cdot k_{d}\left(\mu_{1}+\mu_{2}\right)+k_{d} \mu_{2}}
$$

or rewritten in a more interpretable form as a function of both the natural pulsation $\omega_{n}$ (or natural frequency $f_{n}=\frac{\omega_{n}}{2 \pi}$ ), and the damping factor $\zeta$ as

$$
\begin{array}{r}
L(z)=\frac{2 \zeta \omega_{n} \cdot\left(1-z^{-1}\right)+\omega_{n}^{2}}{\left(1-z^{-1}\right)^{2}+2 \zeta \omega_{n} \cdot\left(1-z^{-1}\right)+\omega_{n}^{2}} \\
\text { with: } \quad\left(\omega_{n} T\right)^{2}=\frac{k_{d} \mu_{2}}{1-k_{d} \mu_{1}} \\
2 \zeta \omega_{n} T=\frac{\left(\mu_{1}-\mu_{2}\right) k_{d}}{1-k_{d} \mu_{1}}
\end{array}
$$

\footnotetext{
${ }^{1} L(z)$ is the same in [1] with $\beta_{d}=\frac{k_{d}}{1-k_{d}, \mu_{1}}$, but differs slightly from the closed-loop transfer function of a 2nd-order digital PLL [30,31], due to the additional branch in dashed line in Fig. 1.
} 
And from (28) and (29), one given couple $\left(\omega_{n}, \zeta\right)$ of the second-order low-pass transfer function can be obtained by tuning $\left(\mu_{1}, \mu_{2}\right)$ as

$$
\begin{aligned}
& \mu_{1}=\frac{1}{k_{d}} \cdot \frac{\left(\omega_{n} T\right)^{2}+2 \zeta \omega_{n} T}{1+\left(\omega_{n} T\right)^{2}+2 \zeta \omega_{n} T} \\
& \mu_{2}=\frac{1}{k_{d}} \cdot \frac{\left(\omega_{n} T\right)^{2}}{1+\left(\omega_{n} T\right)^{2}+2 \zeta \omega_{n} T}
\end{aligned}
$$

The strict stability conditions of $L(z)$ in (26) or (27) versus $\left(\mu_{1}, \mu_{2}\right)$ are given in [2], but if we impose that $0<\omega_{n}<+\infty$ and $0<\zeta<+\infty$ in order to preserve a physical meaning, we deduce from (30)\&(31) that $0<\mu_{2}<\mu_{1}<1 / k_{d}$. We can rewrite $L(z)$ in the frequency-domain, by making $z=e^{p T}$, with $p=j 2 \pi f$, and $f$ is the frequency variable. Fig. 2 plots the modulus of the resulting function, $L\left(e^{j 2 \pi f T}\right)$. Assuming slow reaction of the loop during one OFDM symbol $T$ (i.e. $f_{n} . T \ll 1$ ) and for Low Frequency $(\mathrm{LF})$ region $\left(i . e\right.$. for $f T \ll 1$, using the approximation $z^{-1} \approx 1-p . T$ ), the transfer function of the digital loop in (27) is close to the second-order low-pass transfer function of the analog PLL (i.e. with an active analog lead-lag loop filter, see [33], ch II):

$$
L\left(e^{p T}\right) \approx \frac{2 \zeta \omega_{n} p+\omega_{n}^{2}}{p^{2}+2 \zeta \omega_{n} p+\omega_{n}^{2}}
$$

\subsubsection{Mean Squared Error analysis}

The estimator is unbiased since the CA estimation error $\boldsymbol{\epsilon}_{(n)}$ defined in (20) is zeromean (see (24)). Our aim is to compute the estimation error variance as

$$
\sigma_{\varepsilon}^{2} \stackrel{\text { def }}{=} \frac{1}{L} . E\left\{\boldsymbol{\epsilon}_{(n)}^{H} \boldsymbol{\epsilon}_{(n)}\right\}=\sigma_{\varepsilon \alpha}^{2}+\sigma_{\varepsilon N}^{2}
$$

where $\sigma_{\varepsilon \alpha}^{2}$ is the dynamic error variance, due to the variation of the process $\alpha$, and $\sigma_{\varepsilon N}^{2}$ is the static error variance, due to the additive thermal noise. According to (24) and (20), error $\boldsymbol{\epsilon}_{(n)}$ can be expressed in the Z-domain by $\boldsymbol{\epsilon}(z)=(1-L(z)) \cdot \boldsymbol{\alpha}(z)-k_{d}^{-1} \cdot L(z) \cdot \boldsymbol{N}(z)$. Then, the two components of variance $\sigma_{\varepsilon}^{2}$ can be easily expressed in the frequency- 


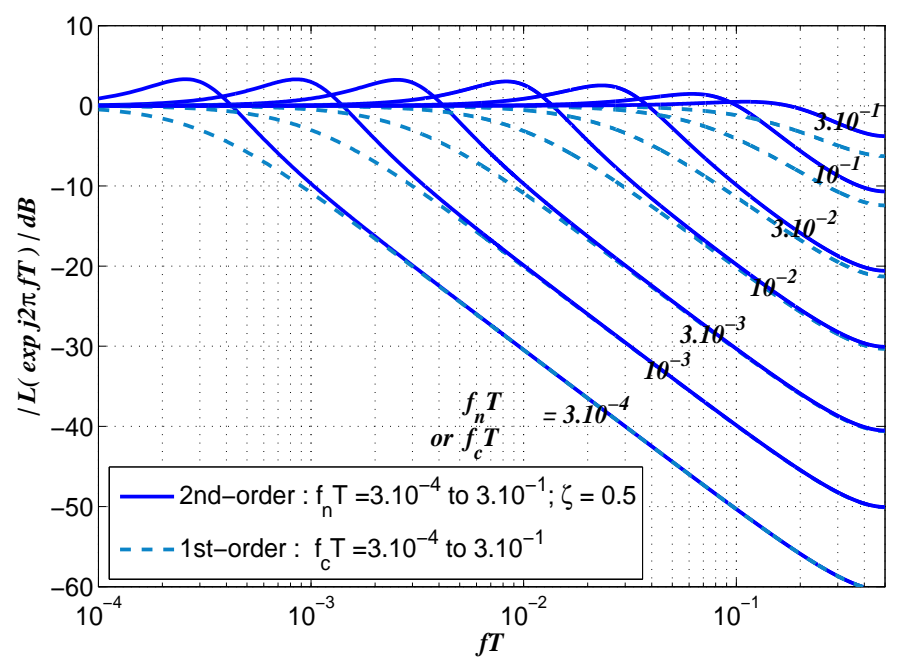

Figure 2: Transfer function $L\left(z=e^{j 2 \pi f T}\right)$ versus normalized frequency $f T$ for the 2nd-order LS-CATL (continuous line) with various normalized natural frequencies $f_{n} T=3 \cdot 10^{-4}$ to $=3 \cdot 10^{-1}$ and a damping factor $\zeta=\frac{1}{2}$, and for a 1st-order LS-CATL (dashed line) with various normalized cut-off frequencies $f_{c} T=$ $3.10^{-4}$ to $=3 \cdot 10^{-1}$.

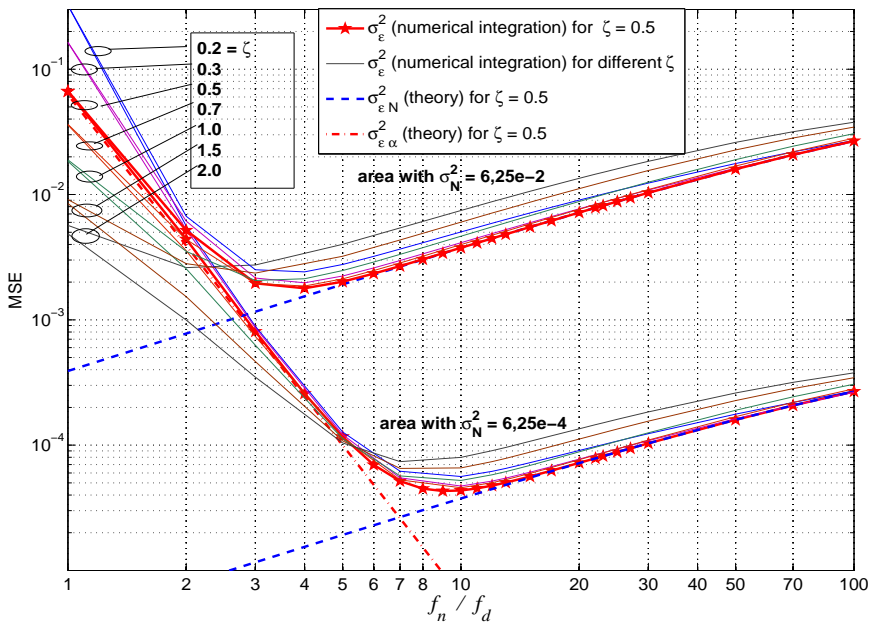

Figure 3: Network of curves of global $\sigma_{\varepsilon}^{2}=\sigma_{\varepsilon \alpha}^{2}+\sigma_{\varepsilon N}^{2}$ (continuous line) versus $f_{n} / f_{d}$ (for a fixed $f_{d} T=$ $1.10^{-3}$ ) for the second-order LS-CATL with various damping factors $\zeta=0.2,0.3,0.5,0.7,1,1.5,2$ computed numerically from (34) assuming Rayleigh-Jakes model (with $\sigma_{\alpha}^{2}=1, L=6$ ), and from (35) assuming $k_{d}^{2}=1$ and $\sigma_{N}^{2}=6,25 \cdot 10^{-2}$ (top of the figure) or $\sigma_{N}^{2}=6,25 \cdot 10^{-4}$ (bottom of the figure). Theoretical reference (dashed line) given from closed-form expressions (36)\&(38) for $\sigma_{\varepsilon N}^{2}$, and from (42) for $\sigma_{\varepsilon \alpha}^{2}$

domain, as is traditionally done when analyzing the tracking performance of a PLL ([33]), or of a predictive LMS estimator ([28]). The component $\sigma_{\varepsilon \alpha}^{2}$ results from the 
high-pass filtering $(1-L(z))$ of the CAs $\boldsymbol{\alpha}_{(n)}$ :

$$
\sigma_{\varepsilon \alpha}^{2}=\int_{-\frac{1}{2 T}}^{+\frac{1}{2 T}} \Gamma_{\alpha}(f) \cdot\left|1-L\left(e^{j 2 \pi f T}\right)\right|^{2} d f
$$

with $\Gamma_{\alpha}(f)=\frac{1}{L} \cdot \sum_{l=1}^{L} \Gamma_{\alpha_{l}}(f)$ where $\Gamma_{\alpha_{l}}$ is the Power Spectrum Density (PSD) of $\alpha_{l}$. The component $\sigma_{\varepsilon N}^{2}$ results from the low-pass filtering $\left(-k_{d}^{-1} . L(z)\right)$ of the input loop noise $N_{(n)}$ :

$$
\sigma_{\varepsilon N}^{2}=\int_{-\frac{1}{2 T}}^{+\frac{1}{2 T}} \Gamma_{N}(f) \cdot \frac{1}{k_{d}^{2}} \cdot\left|L\left(e^{j 2 \pi f T}\right)\right|^{2} d f
$$

with $\Gamma_{N}(f)=\frac{1}{L} \cdot \sum_{l=1}^{L} \Gamma_{N_{l}}(f)$ where $\Gamma_{N_{l}}$ is the PSD of $N_{l}$.

The couple $\left(f_{n}, \zeta\right)$ has to be properly chosen for a good trade-off between the gain in tracking ability and the reduction in loop noise, for a given SNR and $f_{d} T$ scenario. Fig. 3 gives results obtained by numerical integration for $\sigma_{\varepsilon}^{2}$ assuming a "Rayleigh-Jakes" model for the CA dynamic, and a (temporally uncorrelated) input loop noise with two different variances $\sigma_{N}^{2}$. It is shown that fixing $\zeta=\frac{1}{2}$ and varying $f_{n}$ can be a strategy to obtain the best minimum of $\sigma_{\varepsilon}^{2}$. Our objective now is to give some approximate closed-form expressions for $\sigma_{\varepsilon \alpha}^{2}$ and $\sigma_{\varepsilon N}^{2}$, especially for $\zeta=\frac{1}{2}$, approximately.

Static error variance $\sigma_{\varepsilon N}^{2}$. using the whiteness of $N_{l(n)}$ with the PSD $\Gamma_{N}(f)=\sigma_{N}^{2} T$, the equation (35) reduces to

$$
\sigma_{\varepsilon N}^{2}=\frac{\sigma_{N}^{2}}{k_{d}^{2}} \cdot B_{L}
$$

where $B_{L}$ is the (double-sided normalized) noise equivalent bandwidth of the system:

$$
B_{L}=T \times \int_{-\frac{1}{2 T}}^{+\frac{1}{2 T}}\left|L\left(e^{j 2 \pi f T}\right)\right|^{2} d f
$$

An exact analytical expression of $B_{L}$ is derived for the exact second-order loop ((26) or (27)) from the method presented by R. Winkelstein in [34], resulting in

$$
B_{L}=\frac{\left[8 \zeta^{2}+2\right]\left(\omega_{n} T\right)+6 \zeta\left(\omega_{n} T\right)^{2}+\left(\omega_{n} T\right)^{3}}{8 \zeta+\left[8 \zeta^{2}+4\right] \cdot\left(\omega_{n} T\right)+6 \zeta \cdot\left(\omega_{n} T\right)^{2}+\left(\omega_{n} T\right)^{3}}
$$


If $f_{n} . T \ll 1$, we can use the approximation (39) which coincides (see [33], ch. III) with the noise equivalent bandwidth of the usual analog second-order PLL given in (32):

$$
B_{L} \approx 2 \pi f_{n} T \cdot\left(\zeta+\frac{1}{4 \zeta}\right)
$$

Dynamic error variance $\sigma_{\varepsilon \alpha}^{2}$. the dynamic error variance depends on the Doppler spectrum $\Gamma_{\alpha}(f)$ and on $\left|1-L\left(e^{j 2 \pi f T}\right)\right|^{2}$ via the integral form (34). According to (32), the squared modulus of the error transfer function of the second-order loop is

$$
\left|1-L\left(e^{j 2 \pi f T}\right)\right|^{2} \approx \frac{f^{4}}{f_{n}^{4}-f^{2} f_{n}^{2} \cdot\left(2-4 \zeta^{2}\right)+f^{4}} \text { for } f \ll 1 / T
$$

On the other hand, the Doppler spectrum for the "Rayleigh-Jakes" model (4), $\Gamma_{\alpha}(f)=$ $\frac{\sigma_{\alpha}^{2} / L}{\pi f_{d} \sqrt{1-\left(\frac{f}{f_{d}}\right)^{2}}}$ for $\left.f \in\right]-f_{d} ;+f_{d}[$, has a bounded support. Therefore, good tracking will require that the natural frequency of the second-order loop $f_{n}$ be greater than $f_{d}$. Then, we can deduce that only the LF part of the function $\left|1-L\left(e^{j 2 \pi f T}\right)\right|^{2}$ is used in the integral (34), and we can use the LF approximation $\left|1-L\left(e^{j 2 \pi f T}\right)\right|^{2} \approx\left(\frac{f}{f_{n}}\right)^{4}$ for $f \leq f_{d} \ll f_{n} \ll 1 / T$. This approximation is still accurate for $f \approx f_{n}$ for the special case $\zeta \approx \frac{1}{2}$ (see $\left.(40)\right)$. It results that the CA dynamic error variance $\sigma_{\varepsilon \alpha}^{2}$ in (34) can finally be approximated (for $f_{d}<f_{n} \ll 1 / T$, and $\zeta \approx \frac{1}{2}$ ) by

$$
\sigma_{\varepsilon \alpha}^{2} \approx \int_{-f_{d}}^{+f_{d}} \Gamma_{\alpha}(f) \cdot\left(\frac{f}{f_{n}}\right)^{4} d f
$$

For the "Rayleigh-Jakes" model, a variable change $\cos \theta=\left(f / f_{d}\right)$ permits us to evaluate (41) analytically as

$$
\sigma_{\varepsilon \alpha}^{2}(\text { Jakes }) \approx\left(\frac{3}{8}\right) \cdot\left(\frac{f_{d}}{f_{n}}\right)^{4} \cdot \frac{\sigma_{\alpha}^{2}}{L}
$$

Optimal natural frequency. the dynamic component $\sigma_{\varepsilon \alpha}^{2}$ decreases proportionally to the 4 th power of $f_{n}$ according to (42), whereas the static component $\sigma_{\varepsilon N}^{2}$ increases as a function of $f_{n}$, according to (36)\&(39). The component $\sigma_{\varepsilon \alpha}^{2}$ (respectively $\sigma_{\varepsilon N}^{2}$ ) is 
the dominant part of the global $\sigma_{\varepsilon}^{2}$ in the low (respectively large) $f_{n} / f_{d}$ region, as seen in dashed line in Fig. 3. Now, if we fix $\zeta$ (around $\frac{1}{2}$ ), we can calculate the natural frequency $f_{n}$ that permits us (for $f_{d}<f_{n} \ll 1 / T$ ) to minimize the global MSE $\sigma_{\varepsilon}^{2}$ in (33), by searching the zero of the derivative of $\sigma_{\varepsilon}^{2}$ in (33) using (36)\&(39)\&(42):

$$
\left(\frac{f_{n}}{f_{d}}\right)(\text { Jakes })=\left(\frac{3}{4} \cdot \frac{1}{\pi} \cdot \frac{1}{\left(\zeta+\frac{1}{4 \zeta}\right)} \cdot \frac{1}{f_{d} T} \cdot \frac{\sigma_{\alpha}^{2} / L}{\sigma_{N}^{2} / k_{d}^{2}}\right)^{\frac{1}{5}}
$$

The closed-form expression of the corresponding optimal MSE results in

$$
\begin{gathered}
\sigma_{\varepsilon}^{2}(\text { Jakes })=\lambda \cdot\left(\frac{\sigma_{\alpha}^{2}}{L}\right)^{\frac{1}{5}} \cdot\left(\frac{\sigma_{N}^{2}}{k_{d}^{2}} \cdot f_{d} T\right)^{4 / 5} \\
\text { with } \lambda=\frac{15}{8} \cdot\left[\left(\zeta+\frac{1}{4 \zeta}\right) \cdot \frac{4 \pi}{3}\right]^{\frac{4}{5}}
\end{gathered}
$$

It is noticeable that the asymptotic performance of the second-order CATL in (44) coincides, for $\zeta=\frac{\sqrt{2}}{2}$ and $L=1$, with that of the second-order Kalman in [24]-eq.(39), derived for the simplified case of single-carrier and single-path channels.

\subsection{Special case of the first-order CATL}

In the special case where $\mu_{2}=0$, the on-line estimation algorithm is reduced to a first-order low-pass filtering of the LS estimator (see (17) and (21)), such that $\hat{\boldsymbol{\alpha}}_{(n \mid n)}=$ $\left(1-\mu_{1}\right) \cdot \hat{\boldsymbol{\alpha}}_{(n-1 \mid n-1)}+\mu_{1} \cdot \boldsymbol{\alpha}_{\mathbf{L S}(n)}$. The transfer function (25) of the system just depends on a cut-off pulsation $\omega_{c}$ (or cut-off frequency $f_{c}=\frac{\omega_{c}}{2 \pi}$ ), and is reduced to

$$
L(z)=\frac{\omega_{c} T}{\left(1-z^{-1}\right)+\omega_{c} T} \quad \text { with }\left(\omega_{c} T\right)=\frac{k_{d} \mu_{1}}{1-k_{d} \mu_{1}}
$$

and then, approximately, (when $f_{c} . T \ll 1$ ) to an analog first-order low-pass transfer function $L\left(e^{p T}\right) \approx \frac{\omega_{c}}{p+\omega_{c}}$, as can be seen in Fig. 2. We have from (46) that $\mu_{1}=\frac{2 \pi f_{c} T}{1+2 \pi f_{c} T}$. The noise-equivalent bandwidth (37) becomes $B_{L 1}=\frac{2 \pi f_{C} T}{2+2 \pi f_{c} T}$, and can be approxi- 
mated by $B_{L 1} \approx \pi f_{c} T$ when $f_{c} T \ll 1$. As seen in (46) that $\left|1-L\left(e^{j 2 \pi f T}\right)\right|^{2} \approx\left(\frac{f}{f_{c}}\right)^{2}$ for $f \leq f_{d} \leq f_{c} \ll 1 / T$, the dynamic error variance $(34)$ is $\sigma_{\varepsilon \alpha}^{2}($ Jakes $) \approx\left(\frac{1}{2}\right) \cdot\left(\frac{f_{d}}{f_{c}}\right)^{2} \cdot \frac{\sigma_{\alpha}^{2}}{L}$. The minimum global MSE (33) is then reduced, for $f_{d}<f_{c} \ll 1 / T$, to

$$
\begin{aligned}
\sigma_{\varepsilon}^{2}(\text { Jakes }) & =\frac{3}{2} \cdot\left(\frac{\sigma_{\alpha}^{2}}{L}\right)^{\frac{1}{3}} \cdot\left(\pi \cdot \frac{\sigma_{N}^{2}}{k_{d}^{2}} \cdot f_{d} T\right)^{2 / 3} \\
\text { obtained for }\left(\frac{f_{c}}{f_{d}}\right)(\text { Jakes }) & =\left(\frac{1}{\pi} \cdot \frac{1}{f_{d} T} \cdot \frac{\sigma_{\alpha}^{2} / L}{\sigma_{N}^{2} / k_{d}^{2}}\right)^{\frac{1}{3}}
\end{aligned}
$$

It is noticeable that formula (47) of the first-order CATL coincides for $L=1$ with the approximate expression of the asymptotic estimation variance of the AR1-Kalman in [19]-eq.(25), derived for the simplified case of single-carrier and single-path channels.

\section{Simulations}

In this section, the performance of the LS-CATL algorithm is evaluated, first confronted with theoretical analysis and natural references (BCRB and KFs using the same a priori model), and, then, with other algorithms from the literature. By default, we have used a 4QAM-OFDM system, with an FFT size $N=128$ subcarriers, $N_{g}=\frac{N}{8}=16$ samples for the cyclic prefix, and $\frac{1}{T_{s}}=\frac{28}{25} \times 1.25=1.4 \mathrm{MHz}$. These parameters are selected in order to be in concordance with one configuration of the standard Mobile WiMAX Scalable [35], with a subcarrier spacing of $10.94 \mathrm{kHz}$. The number of pilot subcarriers was $N_{p}=6,8,16$, or 32 , corresponding to a distance between pilot subcarriers $L_{f}=22,16,8$, or 4 respectively. The channel model is the Jakes'spectrum Rayleigh channel model with $L=6$ paths and a maximum delay $\tau_{\max }=10$ (expressed as a fraction of $T_{S}$ ) given in $[13,3]$ and recalled in table 2 . The performance is evaluated under a time-varying channel with $f_{d} T \leq 10^{-2}$ (corresponding to a vehicle speed $V_{m} \leq 52.5 \mathrm{~km} / \mathrm{h}$ for $f_{c}=2 \mathrm{GHz}$ ), with a default value $f_{d} T=10^{-3}$. 


\begin{tabular}{ccccccc}
\hline Path number $l$ & 1 & 2 & 3 & 4 & 5 & 6 \\
\hline \hline$\sigma_{\alpha l}^{2} / \sigma_{\alpha}^{2}(d B)$ & -3 & 0 & -2 & -6 & -8 & -10 \\
\hline$\tau_{l}$ & 0 & 1 & 2 & 3 & 4 & 10 \\
\hline
\end{tabular}

Table 2: Average powers and (normalized) delays of the channel

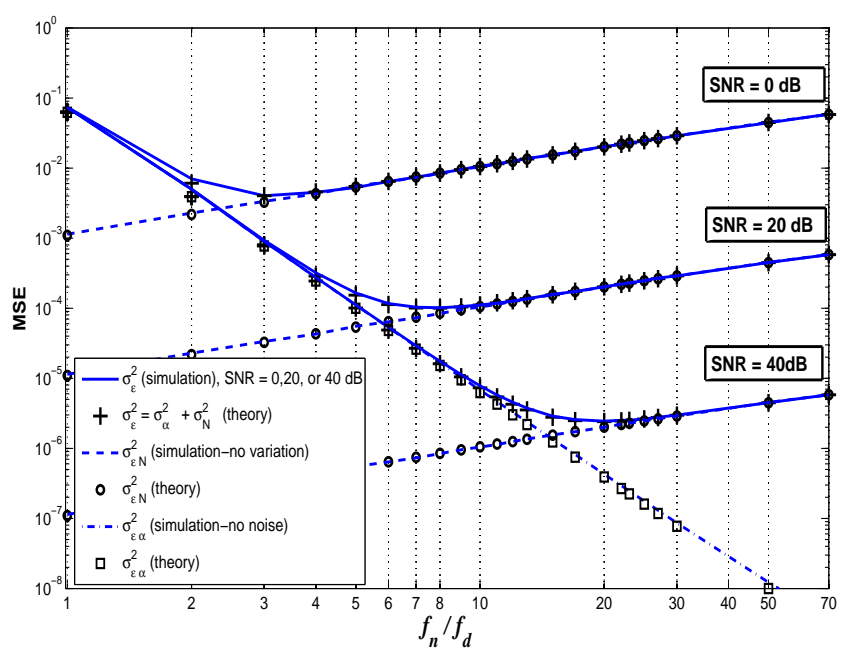

Figure 4: Comparison between simulated and theoretical MSEs vs $f_{n} T$ for $f_{d} T=10^{-3}, \zeta=0.5$, SNR $=0$, 20 , or $40 \mathrm{~dB}, N_{p}=16$ for the proposed 2nd-order LS-CATL algorithm. Theoretical values are given from (36)\&(38) for $\sigma_{\varepsilon N}^{2}$, and from (42) for $\sigma_{\varepsilon \alpha}^{2}$.

\subsection{Confrontation with theory and with KF-based reference algorithms}

\subsubsection{Confrontation with theoretical analysis versus $f_{n} T$}

Fig. 4 gives a comparison between simulated and theoretical error variances versus $f_{n} T$ for $f_{d} T=10^{-3}$, and SNR $=0,20$, or $40 \mathrm{~dB}$ for the proposed 2nd-order (LS-CATL) algorithm, with $N_{p}=16$ pilot subcarriers. The simulated dynamic error variance $\sigma_{\varepsilon \alpha}^{2}$ was measured by forcing the noise $\mathbf{w}_{\mathbf{p}(n)}$ to zero, whereas the simulated static error variance $\sigma_{\varepsilon N}^{2}$ was measured by maintaining the CAs of the paths to constant values equal to their standard deviations $\sigma_{l}$. First of all, we can observe that all the theoretical curves are very close to the simulated ones. Therefore, the abscissa to the minimum of the simulated MSE $\sigma_{\varepsilon}^{2}$ corresponds very well to the theoretical optimal natural frequency (in (43), such that $f_{n} / f_{d}$ (Jakes) $=3,7.4$ and 18.7 respectively for $S N R=0$, 20 , and $40 \mathrm{~dB}$, with $N_{p}=16$ ). It is interesting to note that there is a large range around 
the optimal natural frequency for which the MSE remains very close to the minimum value (for any SNR). Hence, the tuning of the natural frequency of the loop coefficients does not need to be very accurate.

For the rest of the section, we will use the parameters that yield around the minimum possible MSE for the various algorithms. Tables 3(a) and 3(b) give the CATL parameters used for $f_{d} T=10^{-3}$ and $f_{d} T=10^{-2}$, with $N_{p}=16$ pilot subcarriers.

\begin{tabular}{cccccccccc}
\hline$S N R(d B)$ & 0 & 5 & 10 & 15 & 20 & 25 & 30 & 35 & 40 \\
\hline \hline$f_{n} / f_{d}$ & 3 & 4 & 5 & 6 & 8 & 10 & 12 & 15 & 20 \\
(theory $(43))$ & 3 & 3.7 & 4.7 & 5.9 & 7.4 & 9.4 & 11.8 & 14.8 & 18.7 \\
\hline$f_{c} / f_{d}$ & 7 & 10 & 15 & 22 & 34 & 50 & 80 & 130 & 200 \\
(theory $(48))$ & 6.7 & 9.9 & 14.5 & 21.2 & 31.2 & 45.7 & 67.1 & 98.5 & 145 \\
\hline
\end{tabular}

(a) for $f_{d} T=10^{-3}$

\begin{tabular}{cccccccccc}
\hline$S N R(d B)$ & 0 & 5 & 10 & 15 & 20 & 25 & 30 & 35 & 40 \\
\hline \hline$f_{n} / f_{d}$ & 2 & 2.5 & 3 & 4 & 5 & 6 & 8 & 12 & 15 \\
(theory (43)) & 1.9 & 2.4 & 3 & 3.7 & 4.7 & 5.9 & 7.4 & 9.4 & 11.8 \\
\hline$f_{c} / f_{d}$ & 3 & 5 & 7 & 10 & 24 & 30 & 50 & 90 & 400 \\
\hline
\end{tabular}

(b) for $f_{d} T=10^{-2}$

Table 3: Loop parameters $f_{n} / f_{d}$ (2nd-order) and $f_{c} / f_{d}$ (1st-order), for $N_{p}=16$ and $f_{d} T=10^{-3}, 10^{-2}$.

\subsubsection{Comparison with KFs using the same a priori knowledge and parametric model}

We now compare the asymptotic performances to those obtained with two KFs directly derived from our OFDM parametric channel modeling-based estimation problem defined in section 2.3, and using the same a priori knowledge as the proposed LS-CATL. The first one is the AR1-Kalman, which uses an AR1 model to approximate the CA dynamic, and that can be found in [1]-section IV for our specific OFDM model (or in $[8]^{2}$ or $[14]^{3}$ after slight adaptations). But, we also consider as a reference the Or2-Kalman, a Kalman based on a second-order model to better approximate the trend

\footnotetext{
${ }^{2}$ considering in [8] the Kalman-(forward-only)-initial estimation based on pilots. More precisely [8] is the Time-Domain channel estimator that estimates the discrete-time impulse response including both physical channel (CAs at known positions $\boldsymbol{\tau}$ ) and receive filter.

3 adapted for a pilot-aided mode and one polynomial coefficient.
} 
behaviour of the CAs, as in our second-order CATL. It is a kind of extension for multiple carriers and multipath channel of the steady-state version of the KF in [24]. Details about the design of these KFs are given in Appendix A, and table 4 gives the values of the parameters used, yielding around the minimum possible MSE.

\begin{tabular}{ccccc}
\hline & \multicolumn{2}{c}{$f_{d} T=10^{-3}$} & \multicolumn{2}{c}{$f_{d} T=10^{-2}$} \\
& AR1-Kalman & Or2-Kalman & AR1-Kalman & Or2-Kalman \\
\hline \hline$\beta$ & 0 & 0.9992 & 0 & 0.98 \\
$\gamma$ & 0.9996 & 0.9978 & 0.9921 & 0.9975 \\
\hline$\varepsilon$ & $4.10^{-4}$ & $9.10^{-6}$ & $8.10^{-3}$ & $8.10^{-4}$ \\
\hline
\end{tabular}

Table 4: Parameters $(\beta, \gamma)$ used (and related $\varepsilon$ ) for the AR1-Kalman and Or2-Kalman, for $f_{d} T=10^{-3}, 10^{-2}$

Fig. 5(a) and Fig. 5(b) show the evolution of MSE versus SNR, respectively for $f_{d} T=10^{-3}$ and $f_{d} T=10^{-2}$. First of all, the MSE of the proposed 2nd-order LSCATL algorithm is very close to that obtained by the Or2-Kalman algorithm. Likewise, the MSE of the proposed 1st-order LS-CATL algorithm is very close to that obtained by the AR1-Kalman algorithm. It is, therefore, gratifying to verify that the reduced complexity proposed algorithm exhibits almost the same asymptotic variance as the reference Kalman algorithm, for a same model order. It was our desired objective as discussed in the introduction, motivated by some works about phase estimation [31, 32] or about CA estimation in single-carrier flat fading channel [24](ch. 4.1)[25, 26]. Indeed, the authors of these papers have previously proved that PLL, or time-invariant CATL-based filters, can be interpreted, if a satisfactory dynamic model is available, as forms of KF in steady-state mode, with equivalent MSE asymptotic performance.

Secondly, we observe that the performance of the (well-tuned) AR1-Kalman, despite its complexity, does not reach the BRCB in case of slow to moderate channel variation (more notable for $f_{d} T=10^{-3}$ than for $f_{d} T=10^{-2}$ ). We can also incidentally remark an additional degradation (in dashed line), if the AR1-parameter is tuned by the standard correlation matching criterion as in $[8,14]$ (i.e. with $\varepsilon=0$ in (A.3), Appendix A) instead of a minimum variance criterion. This last point corroborates the recent results established in $[18,19]$. 
On the other hand, with a second-order loop (or an Or2-Kalman), the MSE becomes closer to the BCRB (obtained from [6], see also [2]-ch IV). This point reveals the advantage of a second-order loop versus a first-order loop (i.e. with $\mu_{2}=0$ ) in slow to moderate fading scenarios. It allows a higher decrease in the MSE that is proportional to the $\frac{4}{5}$ power of the SNR (in full agreement with the theory (44)), versus the $\frac{2}{3}$ power for the first-order algorithms ((47) and [19]).

Fig. 6 shows the evolution of the Bit Error Rate (BER) in the case of 4-QAM, 16-QAM and 64-QAM modulations for the previous channel estimators completed by a Zero-Forcing (ZF) frequency-domain equalizer. The channel frequency response is previously estimated from the CA estimates $\hat{\boldsymbol{\alpha}}_{(n)}$ by $\hat{\mathcal{H}}_{(n)}=\mathbf{F} \hat{\boldsymbol{\alpha}}_{(n)}$. The BER results agree with the previous MSE results, but with a lower difference between the curves due to the decision process. Hence, the performance with our 2nd-order LS-CATL algorithm is the same in terms of BER as with the Or2-Kalman, and is slightly better (for low SNR regions) than with the AR1-Kalman (and then the 1st-order LS-CATL). The BER performances are close to those found with a ZF equalizer using perfect channel knowledge.

\subsection{BER comparison with other literature algorithms}

Fig. 7 shows for $f_{d} T=10^{-3}$ the BER performances of the proposed LS-CATL algorithm, using a ZF equalizer and a 4-QAM modulation. Also shown are the performances of the previous AR1-Kalman and Or2-Kalman references, together with three estimators that have been suggested in the literature and discussed in the introduction: the "conventional" LS(FD)-LPI [4], the Kalman(FD)-LPI [7] $]^{4}$, and the Kalman-EIG [9] ${ }^{5}$. Note that the latter requires the availability of the power-delay profile to perform "eigenvalue interpolation" of the channel, unlike the two previous algorithms that performed a "bind" LPI interpolation. It is, first of all, highly noteworthy that a "first-

\footnotetext{
${ }^{4}$ the per-subchannel KF has been adapted to our pilot scheme since [7] considered a full block of pilots

${ }^{5}$ corresponds to the initial (pilot-based) channel estimator in [9]
} 


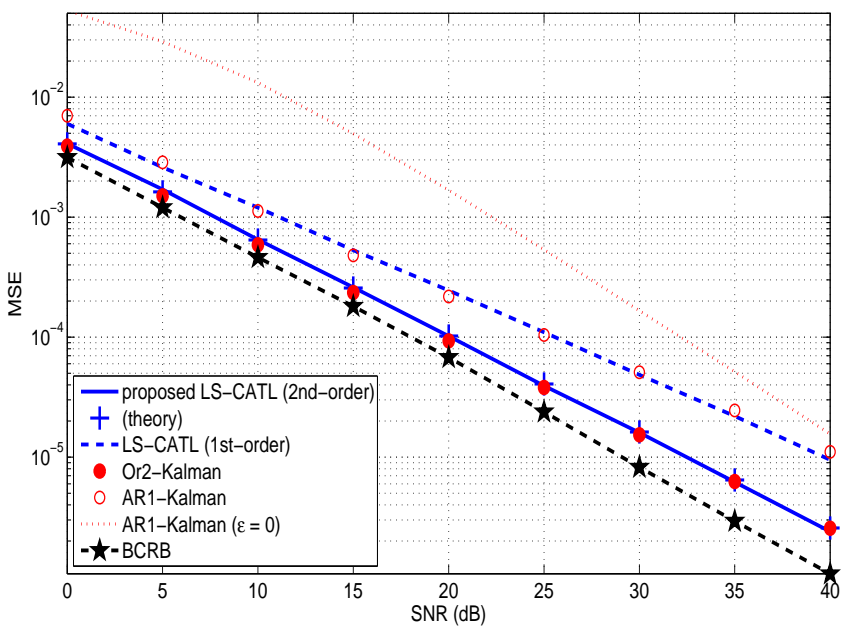

(a) $f_{d} T=10^{-3}$

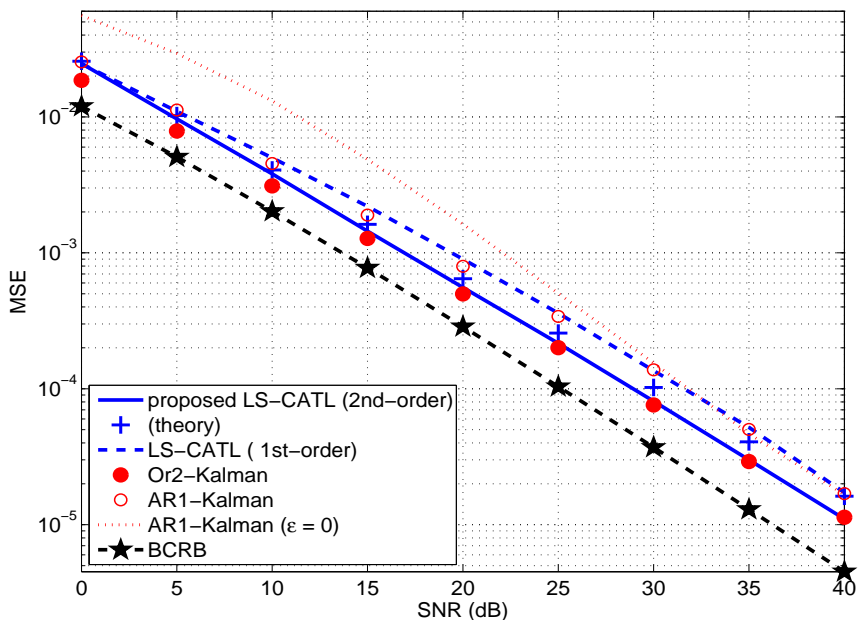

(b) $f_{d} T=10^{-2}$

Figure 5: MSE vs SNR for $f_{d} T=10^{-3}$ (a) and $f_{d} T=10^{-2}$ (b), with $N_{p}=16$

category" of algorithms (proposed CATL, Or2-Kalman, AR1-Kalman and KalmanEIG) greatly outperforms the conventional LS(FD)-LPI method, even if the latter uses a greater number ${ }^{6}$ of pilot subcarriers $\left(N_{p}=64\right.$ versus only $\left.N_{p}=8\right)$. These results per-

\footnotetext{
${ }^{6}$ for the LPI interpolation, the number of pilots must actually fulfill $N_{p} \geq 10$ here if we impose to satisfy the sampling theorem (with then a sampling rate in frequency-domain $L_{f}$ such that [10]: $\frac{N}{L_{f}} \geq \frac{\tau_{\max }}{T_{s}}=10$ ).
} 


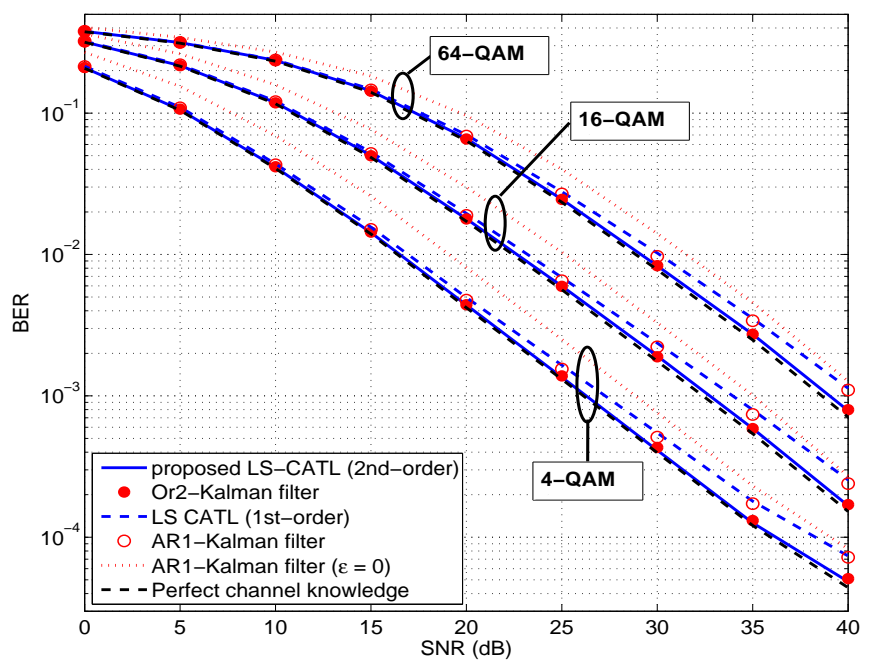

Figure 6: BER comparison for 4-QAM, 16-QAM or 64-QAM modulations, $f_{d} T=10^{-3}$ and $N_{p}=8$

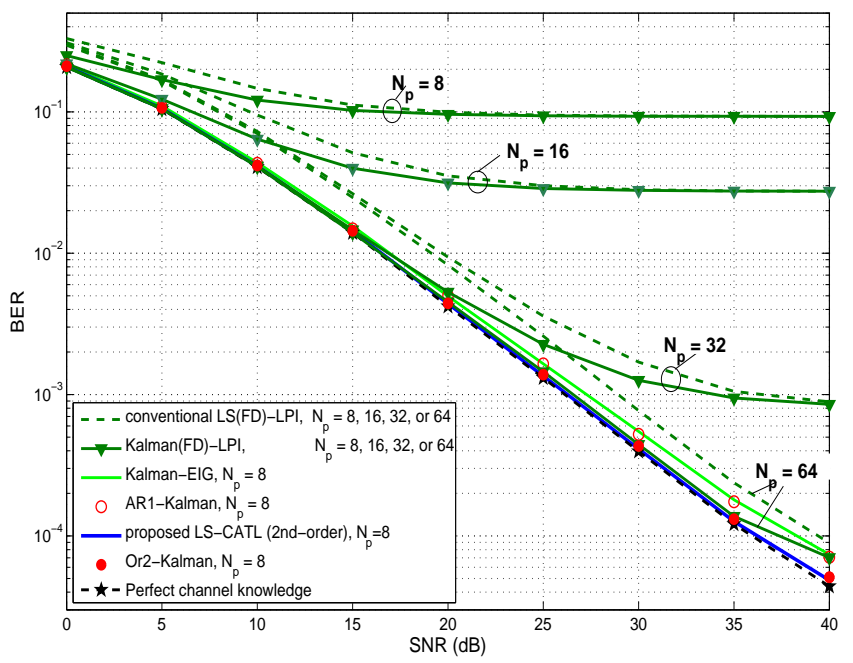

Figure 7: BER comparison with various literature methods using equal or greater number of pilot subcarriers $N_{p}$ than the proposed method, for $f_{d} T=10^{-3}$

mit us to measure the gain when exploiting time-domain correlation, frequency-domain correlation, as well as knowledge of the delays-related information ("first-category") versus only frequency correlation (conventional).

When the conventional symbol by symbol LS(FD)-LPI method is extended into 
Kalman(FD)-LPI algorithm to improve the estimation of the channel at pilot frequency positions (before performing the LPI interpolation in frequency-domain), we can measure the increase in performance due to the use of the past symbols. The benefit of the time-filtering is mainly observed in low SNR regions, and more notable for the lower $f_{d} T$, because of a stronger channel time-correlation. But, the resulting performance still remains far from that of the "first category" of algorithms, unless if $N_{p}=64$ pilot subcarriers are used (i.e. a distance $L_{f}=2$ between two pilot subcarriers). Hence, the availability or the non-availability of the delay-related information is an assumption that influences strongly the channel estimator performance (as discussed more in [2] as well as the effect of an imperfect delay knowledge). We may also note that the BER obtained with the Kalman-EIG is almost the same as with the AR1-Kalman, for a comparable complexity (both algorithms use KF based on the same AR1 state-space model with $N_{c}=6$ dominant eigenvalues tracked in the Kalman-EIG, versus $L=6$ paths CAs in the AR1-Kalman). In conclusion, among the algorithms in the "first category" regarding the asymptotic performance, the proposed LS-CATL algorithm is the one with the lowest complexity, as seen in section 3.3 and table 1 .

\section{Conclusion}

A complex amplitude (CA) estimator of the channel paths over slow to moderate fading channels has been proposed and analyzed. It can be directly useful for either Data Aided or Data Directed single-carrier systems over flat fading channels. Applied to OFDM systems with a comb-type pilot sub-carrier arrangement, it belongs to the class of algorithms that perform the tracking of the CAs of a multipath channel from the information related to path delays. Therefore, it is assumed that an acquisition procedure has already been put into place to calculate path delays. The proposed algorithm is based on a 2nd-order recursive loop, that integrates an error signal created from the pilot-based LS estimates of the CAs. It allows the time-domain correlation of the 
channel to be exploited more simply than the Kalman-based methods, which require matrix inversion at each iteration. Simulation results show that the MSE performance of our 2nd-order algorithm is very close to that of a Kalman estimator based on a 2nd-order approximation of the actual channel. Moreover, our 2nd-order algorithm outperforms the more complex Kalman estimator when the latter is based only on a 1st-order Auto-Regressive model. This emphasizes the advantage of 2nd-order versus 1 st-order methods in the case of slow to moderate fading variation $\left(f_{d} T \leq 10^{-2}\right)$. We have given closed-form expressions of the optimal natural frequency of the loop, and the corresponding minimum MSE (assuming Rayleigh-Jakes channel). We have demonstrated that the MSE of our 2nd-order algorithm decreases proportionally to the $\frac{4}{5}$ power of the SNR, and increases proportionally to the $\frac{4}{5}$ power of the normalized Doppler frequency $f_{d} T$. Moreover, BER comparison through simulation has shown that the proposed algorithm outperforms the basic conventional method based on LPI interpolation in the frequency-domain.

\section{Appendix A. AR1-Kalman (review) and Or2-Kalman filters}

We present two KFs as obvious benchmarks for our specific (parametric channel modeling-based) estimation problem defined in section 2.3. Since exact linear state evolution equation for the Jakes' process is not available, the flat fading CA dynamic has to be approximated in the perspective to use KF (without guarantee of optimality). Let us first introduce the general dynamic model, which will be next declined into AR1 and Or2 models, to approximate the variation of one Jakes'process $\alpha_{l(n)}$ by $\tilde{\alpha}_{l(n)}$ :

$$
\begin{aligned}
& \tilde{\alpha}_{l(n)}=\gamma \cdot \tilde{\alpha}_{l(n-1)}+\delta_{l(n-1)} \\
& \delta_{l(n)}=\beta \cdot \delta_{l(n-1)}+u_{l(n)}
\end{aligned}
$$

where $\gamma$ and $\beta$ are two positive scalars with values lower or equal to 1 , and $u_{l(n)}$ is zero-mean Gaussian complex circular with a variance $\sigma_{u_{l}}^{2}$. 


\section{Appendix A.1. AR1-Kalman}

The special case of AR1-model corresponds to $\beta=0$ (no drift) and $\gamma<1$. The specific equations of the AR1-Kalman applied to our OFDM parametric model can be found in [1]-section IV. We just want to briefly report here recent results about the choice of $\gamma$ for the "Rayleigh-Jakes" channel estimation. For the AR1 model, the autocorrelation function of the approximated process is $R_{\tilde{\alpha}_{l}}^{(k)} \stackrel{\text { def }}{=} E\left\{\tilde{\alpha}_{l(n)} \cdot \tilde{\alpha}_{l(n-k)}^{*}\right\}=$ $\frac{\sigma_{u_{l}}^{2}}{1-\gamma^{2}} \cdot \gamma^{|k|}$ with then $\gamma=R_{\tilde{\alpha}_{l}}^{(1)} / R_{\tilde{\alpha}_{l}}^{(0)}$. Assuming the same variance for the approximated process and the true process (i.e. $R_{\tilde{\alpha}_{l}}^{(0)}=\sigma_{\alpha_{l}}^{2}$ ), the variance of the state noise is directly fixed by the choice of the AR1-coefficient $\gamma$ as $\sigma_{u_{l}}^{2}=\sigma_{\alpha_{l}}^{2}\left(1-\gamma^{2}\right)$. The standard choice for $\gamma$ becomes $J_{0}\left(2 \pi f_{d} T\right)$ if we impose that the auto-correlation function $R_{\tilde{\alpha}_{l}}^{(k)}$ of the approximate process perfectly matches the Bessel auto-correlation function $R_{\alpha_{l}}^{(k)}$ of the true Jakes' process in (4) for lag $k \in\{0,1\}$ (or for $k \in\{0,1, \ldots, p\}$ for a model with order p). This choice corresponds to a correlation matching (CM) criterion, and is the most often used in the literature $([8,9,14,16,22,23])$. However, imposing the matching of the first two taps $\left(R_{\tilde{\alpha}_{l}}^{(0)}=R_{\alpha_{l}}^{(0)}\right.$ and $\left.R_{\tilde{\alpha}_{l}}^{(1)}=R_{\alpha_{l}}^{(1)}\right)$ for $p=1$ does not ensure a short distance between the two auto-correlation functions, and, even less, the minimum estimation variance of the AR1-Kalman. This is especially true for low $f_{d} T \ll 1$ (see [16], Fig. 1) where the taps for lags 1 and 0 have very close values (since $J_{0}\left(2 \pi f_{d} T \times 1\right) \approx$ $\left.1-\frac{1}{4} \cdot\left(2 \pi f_{d} T\right)^{2} \approx 1=J_{0}\left(2 \pi f_{d} T \times 0\right)\right)$, and then the exponential decay of the AR1 autocorrelation function $R_{\tilde{\alpha}_{l}}^{(k)}=\sigma_{\alpha_{l}}^{2} \cdot \gamma^{|k|}$ is imposed so as to be too slow compared to the Bessel function decay, $\sigma_{\alpha_{l}}^{2} \cdot J_{0}\left(2 \pi f_{d} T \times k\right)$. Thereby, we consider possible lower values for the AR coefficient $\gamma$ as in [18, 19, 21]:

$$
\gamma=\frac{J_{0}\left(2 \pi f_{d} T\right)}{1+\varepsilon} \quad(\text { AR1-Kalman })
$$

where $\varepsilon$ is a very small positive amount $(\ll 1)$. Such a slight change can decrease strongly the estimation variance of the AR1-Kalman as proved recently in $[18,19]$. 


\section{Appendix A.2. Or2-Kalman}

For the second-order model, the coefficients $\gamma$ and $\beta$ are non zero. The special case $\gamma=\beta=1$ corresponds to the IRW model [24], with just one parameter $\left(\sigma_{u_{l}}^{2}\right)$ to be adjusted. But we can keep a more general second-order model with two coefficients $\gamma$ and $\beta$ to be adjusted with values lower and close to one. In this case, the state-noise variance will be fixed versus the 2 coefficients $(\beta, \gamma)$ by $\sigma_{u_{l}}^{2}=\sigma_{\delta_{l}}^{2}\left(1-\beta^{2}\right)$, where $\sigma_{\delta_{l}}^{2}=\sigma_{\alpha_{l}}^{2}\left(1+\gamma^{2}\right)-2 \gamma \cdot R_{\tilde{\alpha}_{l}}^{(1)}$ is the variance of the drift $\delta_{l}$. In order to more easily tune the couple of coefficients in section 4 empirically, we use eq. (A.4) (derived from (A.1)\&(A.2)) that gives $\gamma$ wrt to $\beta$ and the two correlation coefficients $R_{\tilde{\alpha}_{l}}^{(1)}, R_{\tilde{\alpha}_{l}}^{(2)}$, the latter being fixed in (A.5) wrt $f_{d} T$ by $\mathrm{CM}$ criterion (with possible adjustment $\varepsilon \ll 1$ ):

$$
\begin{aligned}
\gamma & =\frac{R_{\tilde{\alpha}_{l}}^{(2)}-\beta R_{\tilde{\alpha}_{l}}^{(1)}}{R_{\tilde{\alpha}_{l}}^{(1)}-\beta \cdot \sigma_{\alpha_{l}}^{2}} \quad(\text { Or2-Kalman }) \\
R_{\tilde{\alpha}_{l}}^{(1)} & =\frac{\sigma_{\alpha_{l}}^{2}}{(1+\varepsilon)} J_{0}\left(2 \pi f_{d} \cdot T\right) \quad \text { and } \quad R_{\tilde{\alpha}_{l}}^{(2)}=\sigma_{\alpha_{l}}^{2} J_{0}\left(2 \pi f_{d} .2 T\right)
\end{aligned}
$$

To design the KF, the multi-path multi-carrier problem and the CAs evolution have to be re-formulated in a state-space model. The state vector is $\boldsymbol{a}_{(n)}=\left[\boldsymbol{a}_{1(n)}^{T}, \boldsymbol{a}_{2(n)}^{T}, \ldots, \boldsymbol{a}_{L(n)}^{T}\right]^{T}$, where $\boldsymbol{a}_{l(n)}=\left[\tilde{\alpha}_{l(n)}, \delta_{l(n)}\right]^{T}$ includes the CA and the drift for path $l$. The state evolution matrix is $\mathbf{M}=\operatorname{blkdiag}\left\{\mathbf{M}_{1}, \ldots, \mathbf{M}_{L}\right\}$, where $\mathbf{M}_{l}=\left[\begin{array}{ll}\gamma & 1 \\ 0 & \beta\end{array}\right]$ for $l=1 \ldots L$, and the statenoise vector is $\mathbf{u}_{(n)}=\left[0, u_{1(n)}, \ldots, 0, u_{L(n)}\right]^{T}$. The observation matrix with size $N_{p} \times 2 L$ is $\mathbf{S}_{(n)}=\mathcal{K}_{(n)} \mathbf{Z}$, where the $L \times 2 L$ matrix $\mathbf{Z}$ is defined by $\mathbf{Z}=\operatorname{blkdiag}\left\{\left[\begin{array}{ll}1 & 0\end{array}\right],\left[\begin{array}{ll}1 & 0\end{array}\right], \ldots,\left[\begin{array}{ll}1 & 0\end{array}\right]\right\}$. Then, the state evolution (A.1)\&(A.2) and the observation (5) become $\boldsymbol{a}_{(n)}=\mathbf{M} \boldsymbol{a}_{(n-1)}+$ $\mathbf{u}_{(n)}$ and $\mathbf{y}_{\mathbf{p}(n)}=\mathbf{S}_{(n)} \boldsymbol{a}_{(n)}+\mathbf{w}_{\mathbf{p}(n)}$ from which the Or2-Kalman can be calculated by standard KF equations [27]:

Time Update Equations:

$$
\begin{aligned}
\hat{\boldsymbol{a}}_{(n \mid n-1)} & =\mathbf{M} \hat{\boldsymbol{a}}_{(n-1 \mid n-1)} \\
\mathbf{P}_{(n \mid n-1)} & =\mathbf{M} \mathbf{P}_{(n-1 \mid n-1)} \mathbf{M}^{H}+\mathbf{U}
\end{aligned}
$$




\section{Measurement Update Equations:}

$$
\begin{aligned}
\mathbf{K}_{(n)} & =\mathbf{P}_{(n \mid n-1)} \mathbf{S}_{(n)}^{H}\left(\mathbf{S}_{(n)} \mathbf{P}_{(n \mid n-1)} \mathbf{S}_{(n)}^{H}+\sigma^{2} \mathbf{I}_{N_{p}}\right)^{-1} \\
\hat{\boldsymbol{a}}_{(n \mid n)} & =\hat{\boldsymbol{a}}_{(n \mid n-1)}+\mathbf{K}_{(n)}\left(\mathbf{y}_{p(n)}-\mathbf{S}_{(n)} \hat{\boldsymbol{a}}_{(n \mid n-1)}\right) \\
\mathbf{P}_{(n \mid n)} & =\mathbf{P}_{(n \mid n-1)}-\mathbf{K}_{(n)} \mathbf{S}_{(n)} \mathbf{P}_{(n \mid n-1)}
\end{aligned}
$$

where $\mathbf{K}_{(n)}$ is the Kalman gain matrix (with size $2 L \times N_{p}$ ) and $\mathbf{U}=\operatorname{diag}\left\{0, \sigma_{u_{1}}^{2}, \ldots, 0, \sigma_{u_{L}}^{2}\right\}$.

\section{Appendix B. Interpretation and Kalman-type formulation of the CATL structure}

We define $\hat{\boldsymbol{\delta}}_{(n+1 \mid n)}$ and $\hat{\boldsymbol{\delta}}_{(n \mid n)}$ as the a priori and a posteriori slope estimates of the CAs, forced to be equal and related to $\boldsymbol{v}_{\mathbf{L a g}(n)}$ by:

$$
\hat{\boldsymbol{\delta}}_{(n \mid n)}=\hat{\boldsymbol{\delta}}_{(n+1 \mid n)}=\mu_{2} \cdot \boldsymbol{v}_{\mathbf{L a g}(n)}
$$

Then, the recursive equations of the CATL (8)-(12) can be rewritten by (8)(9)(B.2)(B.4), with the purpose of estimating at each iteration the a posteriori state vector $\left(\hat{\boldsymbol{\alpha}}_{(n \mid n)}, \hat{\boldsymbol{\delta}}_{(n \mid n)}\right)$, using the a priori (or predicted) estimates $\left(\hat{\boldsymbol{\alpha}}_{(n \mid n-1)}, \hat{\boldsymbol{\delta}}_{(n \mid n-1)}\right)$, by:

\section{Measurement Update Equations}

$$
\begin{aligned}
\boldsymbol{v}_{\boldsymbol{\epsilon}(n)} & =\text { function of }\left\{\mathbf{y}_{\mathbf{p}(n)} ; \hat{\boldsymbol{\alpha}}_{(n \mid n-1)}\right\} \\
\hat{\boldsymbol{\alpha}}_{(n \mid n)} & =\hat{\boldsymbol{\alpha}}_{(n \mid n-1)}+\mu_{1} \cdot \boldsymbol{v}_{\boldsymbol{\epsilon}(n)} \\
\hat{\boldsymbol{\delta}}_{(n \mid n)} & =\hat{\boldsymbol{\delta}}_{(n \mid n-1)}+\mu_{2} \cdot \boldsymbol{v}_{\boldsymbol{\epsilon}(n)}
\end{aligned}
$$

\section{Time Update Equations}

$$
\begin{aligned}
\hat{\boldsymbol{\alpha}}_{(n+1 \mid n)} & =\hat{\boldsymbol{\alpha}}_{(n \mid n)}+\hat{\boldsymbol{\delta}}_{(n \mid n)} \\
\hat{\boldsymbol{\delta}}_{(n+1 \mid n)} & =\hat{\boldsymbol{\delta}}_{(n \mid n)}
\end{aligned}
$$

where (B.2) has replaced (10), using (B.1), and (B.3)\&(B.4) have replaced (11)\&(12), using (9)\&(B.1). As in the KF principle, we show the Measurement Update Equations (correction), and the Time Update Equations (prediction). Actually, the CATL 
equations (9)(B.2)-(B.4) coincide for one given path $l$ with the steady-state equations of the KF in [24] derived under the assumption of a dynamic IRW model for $\alpha_{l}$, but for a simplified single-carrier and single-path channel scenario. The vector $\left(\mu_{1}, \mu_{2}\right)$ in (9)\&(B.2) is the steady-state Kalman gain for this simplified situation. In other words, the more complex Or2-Kalman described in Appendix A-(A.6)-(A.10) would be reduced in steady-state mode to the CATL if the observation model (5) were simplified in $\mathbf{y}_{\mathbf{p}(n)}=\boldsymbol{\alpha}_{(n)}+\mathbf{w}_{\mathbf{p}(n)}$, leading to the simple error signal $\boldsymbol{v}_{\boldsymbol{\epsilon}(n)}=\mathbf{y}_{\mathbf{p}(n)}-\hat{\boldsymbol{\alpha}}_{(n \mid n-1)}$.

\section{References}

[1] L. Ros, H. Hijazi and E.P. Simon "Paths Complex Gain Tracking Algorithms for OFDM Receiver in Slowly-Varying Channels" in Proc. IEEE ISCCSP, pp. 1-6, Limassol, Cyprus, March 2010.

[2] L. Ros, H. Hijazi and E.P. Simon "Complex Amplitudes Tracking Loop for multipath slow fading channel estimation in OFDM systems" in Gipsa-lab research report, accessible on-line, April 2012.

[3] Y. Zhao and A. Huang, "A novel Channel estimation method for OFDM Mobile Communications Systems based on pilot signals and transform domain processing" in Proc. IEEE Vehicular Techno. Conf., Phonix, USA, pp. 2089-2093, May 1997.

[4] M. Hsieh and C. Wei, "Channel estimation for OFDM systems based on combtype pilot arrangement in frequency selective fading channels" in IEEE Trans. Consumer Electron., vol.44, no. 1, pp. 217-225, Feb. 1998.

[5] S. Coleri, M. Ergen, A. Puri and A. Bahia, "Channel estimation techniques based on pilot arrangement in OFDM systems" in IEEE Trans. Broad., vol.48, no. 3, pp. 223-229, Sept 2002. 
[6] H. Hijazi and L. Ros, "Bayesian Cramer-Rao bounds for complex gain parameters estimation of slowly varying Rayleigh channel in OFDM systems" in Elsevier Signal Process., Fast communication, Vol 89, pp. 111-115, Jan. 2009.

[7] W. Chen and R. Zhang, "Kalman filter Channel estimator for OFDM systems in time and frequency-selective fading environment" in Proc. IEEE ICASSP, vol. 4, pp. 17-21, Montreal, Canada, May 2004.

[8] T.Y. Al-Naffouri, "An EM-based Forward-Backward Kalman Filter for the Estimation of Time-Variant Channels in OFDM" in IEEE Trans. Signal Process, vol. 55, no. 7, pp. 3924-3930, July 2007.

[9] M.S. Sohail, T.Y. Al-Naffouri, “An EM-based frequency domain channel estimation algorithm for multi-access OFDM systems" in Elsevier Signal Process., vol. 90, no. 5, pp. 1562-1572, May 2010.

[10] B. Yang, K. B. Letaief, R. S. Cheng and Z. Cao, "Channel estimation for OFDM transmission in multipath fading channels based on parametric channel modeling" in IEEE Trans. Com., vol. 49, no. 3, pp. 467-479, March 2001.

[11] M. Raghavendra, S. Bhashyam, and K. Giridhar, "Exploiting hopping pilots for parametric channel estimation in OFDM systems" in IEEE Signal Process. Lett., vol. 12 , no. 11, pp. 737-740, Nov. 2005.

[12] N. Chen, J. Zhang, and P. Zhang, "Improved Channel Estimation Based on Parametric Channel Approximation Modeling for OFDM Systems" in IEEE Trans. Broad., vol. 54, no. 2, June 2008.

[13] H. Hijazi and L. Ros, "Polynomial estimation of time-varying multi-path gains with intercarrier interference mitigation in OFDM systems" in IEEE Trans. Vehicular Techno., vol. 58, no. 1, pp.140-151, January 2009. 
[14] H. Hijazi and L. Ros, “Joint Data QR-Detection and Kalman Estimation for OFDM Time-varying Rayleigh Channel Complex Gains" in IEEE Trans. Com., vol. 58 , no. 1 , pp. 170-178, January 2010.

[15] E.P. Simon, L. Ros, H. Hijazi, M. Ghogho, "Joint carrier frequency offset and channel estimation for OFDM systems via the EM algorithm in the presence of very high mobility" in IEEE Trans. Signal Process., vol. 60, no. 2, pp. 754-765, Feb. 2012.

[16] C. Komninakis, C. Fragouli, A.H. Sayed, and R.D. Wesel, "Multi-Input MultiOutput Fading Channel Tracking and Equalization Using Kalman Estimation”, in IEEE Trans. Signal Process., vol. 50, no. 5, pp. 1065-1076, May 2002.

[17] Z. Liu, X. Ma, and G. Giannakis, "Space-time coding and Kalman filtering for time-selective fading channels," in IEEE Trans. Com., vol. 50, no. 2, pp. 183-186, Feb. 2002.

[18] A. Barbieri, A. Piemontese, G. Colavolpe, "On the ARMA Approximation for Frequency Channels Described by the Clarke Model with Applications to Kalman-based Receivers", in IEEE Trans. on Wireless Com., vol. 8, no. 2, Feb. 2009.

[19] S. Ghandour-Haidar, L. Ros, J.M. Brossier, "On the Use of first-order Autoregressive Modeling for Rayleigh Flat Fading Channel Estimation with Kalman filter", in Elsevier Signal Process., vol. 92, no. 2, pp. 601-606, Feb. 2012.

[20] W. C. Jakes, Microwave Mobile Communications. IEEE Press, 1983.

[21] K.E. Baddour, N.C. Beaulieu, "Autoregressive modeling for fading channel simulation", in IEEE Trans. Wireless Com., vol. 4, no. 4, pp. 1650-1662, Jul. 2005.

[22] H. Wang and P. Chang, "On verifying the first-order Markovian assumption for 
a Rayleigh fading channel model" in IEEE Trans. Vehicular Techno., vol. 45, pp. 353-357, May 1996.

[23] P. Banelli, R. Cannizzaro, and L. Rugini, “Data-Aided Kalman Tracking for Channel Estimation in Doppler-Affected OFDM Systems" in Proc. IEEE ICASSP, vol. 3, pp. 133-136, Hawaï, USA, April 2007.

[24] L. Ros, and E.P. Simon "Second-order modeling for Rayleigh flat fading channel estimation with Kalman Filter" in Proc. IEEE DSP, pp. 1-6, Corfou, Greece, Jul. 2011.

[25] S. Gazor, "Prediction in LMS-type Additive Algorithms for Smoothly Time Varying Environments" in IEEE Trans. Signal Process., vol. 47, no. 6, pp. 1735-1739, June 1999.

[26] L. Lindbom, A. Ahlen, M. Sternad, and M. Falkenstrom, "Tracking of TimeVarying Mobile Radio Channels-Part II : A Case Study" in IEEE Trans. Com., vol. 50, no. 1, pp. 156-167, Jan. 2002.

[27] S.M. Kay, Fundamentals of Statistical Signal Processing: Estimation Theory. Prentice Hall PTR, 1993.

[28] Q. Nassir, "Wireless Channel Tracking Based on Optimum Predictive LMS" in Wireless Personal Com., vol. 48, no. 4, pp. 511-519, March 2009.

[29] U. Mengali, A.N. D’Andrea, Synchronization Techniques for Digital Receivers (Applications of Communications Theory). PLENUM, 1997.

[30] Y. R. Shayan and T. Le-Ngoc, "All digital phase-locked loop: concepts, design and application" in IEE Proceedings, vol. 136, no. 1, pp. 53-56, February 1989.

[31] A. Patapoutian, "On phase-locked loops and Kalman filters" in IEEE Trans. Com., vol. 47, pp. 670-672, May 1999. 
[32] G. S. Christiansen, "Modeling of a PRML timing loop as a Kalman filter", in Proc. IEEE Globecom, vol. 2, pp. 1157-1161, 1994.

[33] F.M. Gardner, Phaselock Techniques. Wiley, 2nd edition, 1979.

[34] R. Winkelstein, "Closed Form Evaluation of Symmetric Two-Sided Complex Integrals", in TDA Progress Report, no. 42-65, pp. 133-141, July and August 1981.

[35] J.G. Andrews, A. Gosh, R. Muhamed, Fundamentals of WiMAX: Understanding Broadband Wireless Networking.Prentice Hall, 2007. 